Ivan Giorgio · Antonio Culla · Dionisio Del Vescovo

\title{
Multimode vibration control using several piezoelectric transducers shunted with a multiterminal network
}

\begin{abstract}
In this paper a new approach is presented to reduce vibrations for one- and two-dimensional mechanical structures, as beam or thin plates, by means of several piezoelectric transducers shunted with a proper electric network system. The governing equations of the whole system are coupled to each other through the direct and converse piezoelectric effect. More in detail, the mechanical equations are expressed in accordance with the modal theory considering $n$ vibration modes and the electrical equations reduce to the one-dimensional charge equation of electrostatics for each of $n$ considered piezoelectric transducers. In this electromechanical system, a shunting electric device forms an electric subsystem working as multi degrees of freedom (dof's) damped vibration absorber for the mechanical subsystem. Herein, it is introduced a proper transformation of the electric coordinates in order to approximate the governing equations for the whole shunted system with $n$ uncoupled, single mode piezoelectric shunting systems that can be readily damped by the methods reported in literature. A further numerical optimisation problem on the spatial distribution of the piezoelectric elements allows to achieve a better performance. Numerical case studies of two relevant systems, a double clamped beam and a fully clamped plate, allow to take into account issues relative to the proposed approach. Laboratory experiments carried out in real time on a beam clamped at both ends consent to validate the proposed technique.
\end{abstract}

Keywords Vibration control · Shunt damping · Multi-mode control · Multiple piezoelectric transducers

\section{Introduction}

In the last years the employ of structures more and more thin had made arise numerous issues regarding structural vibrations. Hence, the object of this paper is the reduction of flexural vibrations in mechanical structures, as beam and thin plates. To obtain an effective damping of structural vibrations, piezoelectric transducers are employed. This is primarily due to the growing availability of more efficient piezoelectric materials. Smart structures using piezoelectric elements, indeed, are successfully employed in reducing vibration $[1 ; 2 ; 3 ; 4 ; 5 ; 6 ; 7 ; 8 ; 9]$.

In the shunt-damping technique the reduction of structural vibrations is achieved by shunting a piezoelectric transducer with an electric network that acts to increase the mechanical damping. In details, a piezoelectric transducer coupled to mechanical structures can convey the mechanical energy toward the electric network system where it is dissipated.

A classical application of this method is a single resonant piezoelectric shunting system studied in $[10 ; 11]$. The damper is formed by a piezoelectric element shunted with an inductor and a resistor.

University of Rome "Sapienza", Department of Mechanics and Aeronautics

via Eudossiana, 18 - 00184 Rome, Italy

Tel.: +39-06-44585556, Fax: +39-06-484854

E-mail: ivan.giorgio@uniroma1.it 
The external shunt circuit with the inherent piezoelectric capacitance is an $R L C$ circuit. Its natural frequency is imposed equal to one natural frequency of the original mechanical structure by maximising the energy exchange. The resistance role is to maximise the electric dissipation of the energy coming from the mechanical structure. Its main drawback is the requirement of high-value inductors $(10 \div$ $1000 \mathrm{H})$, working at high-voltage. For this reason, passive components are commonly implemented through active circuits which require an external feeding.

Further applications involve one piezoelectric transducer coupled with a multiresonant electric network to damp a set of mechanical modes. Hollkamp's circuit is one of this kind [12]. The shunt network consists of a set of branches whose main is an $R L$ circuit. The other branches are $R L C$ shunts. The number of controlled mechanical modes is equal to the number of the branches. An issue of this technique is the necessity of retuning the circuit when a branch is added. Indeed, in [12] is proposed no closed-form tuning solution.

Moreover, other approaches use multiple piezoelectric transducers by shunting each of them to a proper multiresonant electric network [13]. In practice, to account for the undesired cross influence of the shunt circuits on the mechanical modes to be controlled, a proper fine-tuning is due.

In $[6 ; 14 ; 15 ; 5]$ systems with periodically distributed piezoelectric transducers and modular shunting networks are considered. This approach adopts homogenised continuum modelling and looks for periodic lumped electric systems having, in the continuum limit, the same dynamic behaviour of the mechanical structure to be controlled. The drawback of this "continuum mechanics" approach is the requirement of a high number of piezoelectric elements and complex shunting networks, in order to approach the continuum limit.

The aim of this study is to extend the resonant shunting techniques to control multiple vibration modes with multiple piezoelectric transducers by an electric network which interconnects the whole set of piezoelectric elements. A great attention has been devoted to design the shunting network system, in order to ensure a satisfactory coordination of all piezoelectric transducers so that all mechanical dof's in need of control are damped at the same time. The key idea in this paper is to make the whole shunted system equivalent to a set of independent single resonant piezoelectric shunting systems. Therefore, it is possible to use the widely investigated methods presented in literature. The proposed network is actualised for the laboratory experiments, despite of its passive nature, with an active feedback control.

\section{Model of the structure integrating multiple piezoelectric transducers}

We consider a structure integrating a set of piezoelectric transducers. The transducers are generally piezoelectric patches bonded on the structure. From the electric point of view, each transducer is characterised by two electrodes and can be regarded as a two-terminal port.

Let $w(\mathbf{x}, t)$ be the displacement field of a linear elastic continua with a set of $n_{p}$ piezoelectric transducer. As a first order of approximation, each piezoelectric transducer is, according to Norton's theorem, electrically equivalent to a strain dependent charge generator in parallel with a capacitance $C_{h}$ and a resistance $R_{h}[16]$.

The following partial differential equations describe the motion of the elastic continua coupled with the dynamic equation, implying the charge conservation, of the piezoelectric transducers

$$
\begin{aligned}
& \mathfrak{L}[w(\mathbf{x}, t)]+\frac{\partial}{\partial t} \mathfrak{D}[w(\mathbf{x}, t)]+\mathfrak{M}(\mathbf{x}) \frac{\partial^{2} w(\mathbf{x}, t)}{\partial t^{2}}=f_{d}(\mathbf{x}, t)+\sum_{h=1}^{n_{p}} \mathcal{P}_{h}\left[\wp_{h}(\mathbf{x})\right] \frac{d \psi_{h}(t)}{d t} \\
& \mathcal{Q}_{h}(t)=\frac{d \psi_{h}(t)}{d t}+\frac{1}{R_{h} C_{h}} \psi_{h}(t)+\int_{\mathcal{A}_{h}} \mathcal{P}_{h}[w(\mathbf{x}, t)] d \mathcal{A}_{h} \quad h=1,2, \ldots n_{p}
\end{aligned}
$$

A set of associated boundary and initial conditions must be considered to solve the differential problem. $\mathfrak{L}, \mathfrak{D}$ and $\mathfrak{M}$ are linear homogeneous differential operators, with respect to the spatial coordinate $\mathbf{x}$. They constitute a model of stiffness, viscous damping and mass density respectively of the system. The term $f_{d}(\mathbf{x}, t)$ is the external disturbance load to the structure and the further sum involves that each of the $n_{p}$ piezoelectric transducer applies a forcing input proportional to the time derivative of the flux linkage, i.e. the terminal voltage of the $h$-th transducer. To simplify the theoretical analysis, the second equation of Eqs. (1) is normalised to a unit piezoelectric capacitance. Thus, the electric 
quantities $\psi_{h}$ and $\mathcal{Q}_{h}$ are respectively the product of the flux linkage by the square root of the inherent capacitance and the induced charge divided by the square root of the same capacitance, $C_{h}$. $\mathcal{P}_{h}$ is a linear homogeneous differential operator and $\wp_{h}(\mathbf{x})$ is a spatial function of piezoelectric localisation that takes the value 1 where the piezoelectric element is placed and 0 everywhere else. The third term of the right hand side of this equation is the normalised charge induced by the piezoelectric effect. The region $\mathcal{A}_{h}$ is that occupied by the $h$-th transducer. In ordinary applications, the internal resistance $R_{h}$ is very large and can be neglected.

The displacement, $w$, of the considered system may be expanded in the series

$$
w(\mathbf{x}, t)=\sum_{i} W_{i}(\mathbf{x}) \eta_{i}(t) \quad \text { with } i=1,2, \ldots
$$

$W_{i}(\mathbf{x})$ are the mode shapes of the $i$-th eigenfrequency of the undamped system for null mechanical load and under short circuit condition: $\dot{\psi}_{h}=0$ for each $h$ (the superscript dot denotes the derivative with respect to $t$ ). Therefore, they are the eigenfunctions that are obtained by solving the eigenvalue problem

$$
\mathfrak{L}[W(\mathbf{x})]=\lambda \mathfrak{M}(\mathbf{x}) W(\mathbf{x})
$$

with its associated boundary conditions. The coefficient $\eta_{i}(t)$ is the generalised coordinate describing the response of the $i$-th normal mode.

Considering $n_{m}$ normal modes, the Eqs. (1) may be rewritten as follows

$$
\left\{\begin{array}{r}
\ddot{\boldsymbol{\eta}}+D \dot{\boldsymbol{\eta}}+\Omega^{2} \boldsymbol{\eta}-\Omega \Gamma \dot{\boldsymbol{\psi}}=\boldsymbol{f} \\
\ddot{\boldsymbol{\psi}}+\Xi \dot{\boldsymbol{\psi}}+(\Omega \Gamma)^{T} \dot{\boldsymbol{\eta}}=\boldsymbol{\imath}
\end{array}\right.
$$

with an opportune set of boundary and initial condition. This is a system of $n_{m}+n_{p}$ ordinary differential equations.

Denoting each natural frequency of undamped oscillation under short circuit condition with $\omega_{i}$, the $n_{m} \times n_{m}$ matrix $\Omega$ is defined as $\Omega_{i h}=\omega_{i} \delta_{i h}$ with $\delta_{i h}$ the Kronecker delta. The $n_{m} \times n_{m}$ damping matrix $D$ is given by

$$
D_{i h}=\int_{\mathcal{A}} W_{i}(\mathbf{x}) \mathfrak{D}\left[W_{h}(\mathbf{x})\right] d \mathcal{A}
$$

is symmetric if the operator $\mathfrak{D}$ is self-adjoint. The unit-frequency normalised coupling matrix $\Gamma$ is a $n_{m} \times n_{p}$ matrix whose entries $\Gamma_{i h}$ represent the coupling coefficient between the $i$-th normal mode and the $h$-th piezoelectric transducer and are defined by

$$
\begin{aligned}
\Gamma_{i h} & =1 / \omega_{i} \int_{\mathcal{A}} W_{i}(\mathbf{x}) \mathcal{P}_{h}\left[\wp_{h}(\mathbf{x})\right] d \mathcal{A} \\
& =1 / \omega_{i} \int_{\mathcal{A}} \wp_{h}(\mathbf{x}) \mathcal{P}_{h}\left[W_{i}(\mathbf{x})\right] d \mathcal{A}
\end{aligned}
$$

The $n_{m}$-dimensional vector $\boldsymbol{f}$, representing mode forces, is given by

$$
f_{i}(t)=\int_{\mathcal{A}} W_{i}(\mathbf{x}) f_{d}(\mathbf{x}, t) d \mathcal{A}
$$

The $n_{p} \times n_{p}$ matrix $\Xi$ is defined as $\Xi_{i h}=\left[1 /\left(R_{h} C_{h}\right)\right] \delta_{i h}$. The column $\boldsymbol{\imath}$ represents the $n_{p^{-}}$-dimensional vector of normalised currents flowing through the piezoelectric elements.

\section{An Independent Modal-Space Shunt Damping Technique}

Let us consider $n_{m}=n_{p}=n$, in order to use each electric dof, $\psi_{h}$, to control one modal mechanical dof, $\eta_{i}$.

An undamped system is considered:

$$
\left\{\begin{array}{r}
\ddot{\boldsymbol{\eta}}+\Omega^{2} \boldsymbol{\eta}-\Omega \Gamma \dot{\boldsymbol{\psi}}=\boldsymbol{f} \\
\ddot{\boldsymbol{\psi}}+(\Omega \Gamma)^{T} \dot{\boldsymbol{\eta}}=\boldsymbol{\imath}
\end{array}\right.
$$


Note that a small amount of damping is not relevant to design the shunt network because it does not produce a significant change in the natural mechanical frequencies and modes. The piezoelectric coupling matrix $\Gamma$ must not be a singular matrix in order to avoid lack of controllability and observability. Generally $\Gamma$ is also not diagonal. In Eqs. (8) mechanical and electric dof's are coupled by means the piezoelectric effect. In general Eqs. (8) represent a set of $2 n$ simultaneous linear second-order ordinary differential equations with constant coefficients.

In order to obtain a set of equations equivalent to Eqs. (8), consisting of $n$ single mode piezoelectric shunting systems $[10 ; 11]$, a coordinate transformation is introduced

$$
\psi=U \chi
$$

where $\chi_{k}(t), k=\{1, \ldots n\}$, are generalised electric coordinates and $U$ is a constant orthogonal square matrix referred to as transformation matrix. Next, premultiplying both sides of the second equation by $U^{T}$, the transpose of $U$, and applying the orthogonal properties of $U$, it results

$$
\left\{\begin{array}{r}
\ddot{\boldsymbol{\eta}}+\Omega^{2} \boldsymbol{\eta}-\Omega \mathcal{G} \dot{\chi}=\boldsymbol{f} \\
\ddot{\chi}+(\Omega \mathcal{G})^{T} \dot{\boldsymbol{\eta}}=\boldsymbol{z}
\end{array}\right.
$$

where the matrix $\mathcal{G}=\Gamma U$ is the electro-mechanical coupling matrix in the electric coordinates $\chi_{k}(t)$ and

$$
\boldsymbol{z}=U^{T} \boldsymbol{\imath}
$$

is an n-dimensional vector whose elements are the electric forcing terms associated with the new coordinates $\chi_{k}(t)$. Comparing Eqs. (10) with (8), it is possible to note that the form of the system does not change for the orthogonality imposition of the transformation matrix $U$. Besides, it is clear from equations (10) that if $\mathcal{G}$ were diagonal, recalling that $\Omega$ is diagonal, the uncoupling by means of the coordinate transformation would be reached. Therefore, it would be possible to identify $n$ uncoupled systems of two equations, each pair constituted by a mechanical equation and an electrical one. It means that each component of $\boldsymbol{\chi}$ influences only the corresponding component of $\boldsymbol{\eta}$ and vice versa.

It should be noted that, if $\Gamma$ were diagonal it would be possible to identify immediately $n$ singlemode piezoelectric shunting systems, without recoursing to transformation $U$. But there would be something inefficient with this arrangement. The action of a piezoelectric transducer is local and so, working each of them on only one mechanical dof, the global damping is very weak. In addition, it is difficult to find an optimal pattern of the piezoelectric set that makes $\Gamma$ diagonal.

\subsection{Linear transformation for independent control}

Let $\mathbb{M}_{n}$ denote the vector space of all square matrices of order $n$ over real field $\mathbb{R}$. It is easy to check that given any square matrix $\Gamma$ in $\mathbb{M}_{n}$ and a matrix $U$ belonging to the orthogonal group $\operatorname{Orth}(n)$, the problem of diagonalising $\mathcal{G}=\Gamma U$ admits solution if and only if the rows of $\Gamma$ are mutually perpendicular. If it does not occur, an exact diagonalisation of $\mathcal{G}$ is not feasible, so that a different approach is desirable. Herein it is proposed to find the best transformation matrix $U$ that makes $\mathcal{G}$ approximatively diagonal. Using the point of view of the set theory, let $\mathbb{G}$ be the set of all possible electro-mechanical coupling matrix that is the set of the matrices $\Gamma U$ as $U$ varies over the orthogonal group $\operatorname{Orth}(n)$ as well let $\mathbb{D}_{n}$ be the vector subspace of the diagonal matrices of order $n$. The wanted $U$ is the matrix which identifies the Euclidean distance between these two sets. Such distance $d\left(\mathbb{G}_{n}, \mathbb{D}_{n}\right)$ between the sets $\mathbb{G}$ and $\mathbb{D}_{n}$ is defined as the infimum of all distances between any two of their respective elements, $\Gamma U$ and $D$, and can be expressed as

$$
d\left(\mathbb{G}, \mathbb{D}_{n}\right)=\inf _{U \in \operatorname{Orth}(n)}\left\{\inf _{D \in \mathbb{D}_{n}}\|\Gamma U-D\|\right\}
$$

where the expression inside the curly brackets defines the orthogonal projection of $\Gamma U$ onto $\mathbb{D}_{n}$ and indeed represents the closest diagonal matrix to $\Gamma U$. Thus, for any matrix $U$, there exists a unique matrix $D_{\Gamma U}$ that belongs to $\mathbb{D}_{n}$ that attain the infimum, that is to say a minimum. In particular, it can be written

$$
\epsilon=\min _{D \in \mathbb{D}_{n}}\|\Gamma U-D\|=\left\|\Gamma U-D_{\Gamma U}\right\|
$$


The non-negative quantity $\epsilon$, identified by $\left\|\Gamma U-D_{\Gamma U}\right\|$, represents the error by approximating $\Gamma U$ with a diagonal matrix.

To find the orthogonal projection $D_{\Gamma U}$ for a given matrix $\Gamma$ and any orthogonal matrix $U$, let $\mathcal{B}=\left\{D_{i}: i=1,2, \ldots n\right\}$ be the standard basis of $\mathbb{D}$ consisted of $n$ diagonal matrices with one in the $i i$-th entry and zero elsewhere. Then, since the matrix $D_{\Gamma U}$ can be written in the form

$$
D_{\Gamma U}=\sum_{h=1}^{n} \alpha_{h} D_{h}
$$

the coefficients $\alpha_{h}$ can be obtained by using the orthogonality of the projection $\left(\Gamma U-D_{\Gamma U}\right) \cdot D_{h}=0$ and the orthonormality of the unit matrices of the chosen basis $D_{h} \cdot D_{k}=\delta_{h k}$ to get $\alpha_{h}=\Gamma U \cdot D_{h}$. Thus, the orthogonal projection of $\Gamma U$ over $\mathbb{D}$ is the operation of taking the diagonal part of the matrix $\Gamma U$.

Besides, since the theorem of Weierstrass states that the real valued continuous function of the matrix $U, \epsilon(U)$, assumes a minimum and a maximum value on the compact subset $\operatorname{Orth}(n)$ of $\mathbb{M}_{n}$, it assures the existence of a matrix $\tilde{U}$ in $\operatorname{Orth}(n)$ that attain the infimum of the expression (12). Thus, the above optimisation problem, equivalently expressed in terms of squared distance, becomes

$$
d\left(\mathbb{G}, \mathbb{D}_{n}\right)^{2}=\min _{U \in \operatorname{Orth}(n)}\left\|\Gamma U-D_{\Gamma U}\right\|^{2}
$$

Taking the properties of the standard inner product for granted, one can expand the cost function for the optimisation problem (15) as follows

$$
\begin{aligned}
\left\|\Gamma U-D_{\Gamma U}\right\|^{2} & =\left(\Gamma U-D_{\Gamma U}\right) \cdot\left(\Gamma U-D_{\Gamma U}\right)= \\
& =\Gamma U \cdot \Gamma U-2 \Gamma U \cdot D_{\Gamma U}+D_{\Gamma U} \cdot D_{\Gamma U}
\end{aligned}
$$

Next, being the matrix $U$ orthogonal, it is easy to check that

$$
\Gamma U \cdot \Gamma U=\Gamma \cdot \Gamma
$$

that is to say the norm of coupling matrix $\mathcal{G}=\Gamma U$ does not depend on transformation matrix $U$. Once again taking into account the orthogonality of the projection, it is possible to write

$$
\left(\Gamma U-D_{\Gamma U}\right) \cdot D_{\Gamma U}=0
$$

Therefore, introducing the Eqs. (17) and (18) into Eq. (16), it turns out that

$$
\left\|\Gamma U-D_{\Gamma U}\right\|^{2}=\Gamma \cdot \Gamma-D_{\Gamma U} \cdot D_{\Gamma U}
$$

It is clear from the Eq. (19) that, for any fixed matrix $\Gamma$, the optimisation problem (15) is equivalent to the problem

$$
\max _{U \in \operatorname{Orth}(n)}\left\|D_{\Gamma U}\right\|^{2}
$$

It turns out that any matrix $\mathcal{G}$ is associated with the transfer of power through the piezoelectric transducers between the mechanical dof's, $\eta_{i}(t)$, and the electric dof's, $\chi_{k}(t)$, employed to control vibrations. The relation (17) shows that this power depend only on the matrix $\Gamma$ and therefore the piezoelectric placement. The role of the transformation matrix $U$ is to improve the energy exchange between the two linked systems. In fact, the two optimisation problems (15) and (20) are equivalent as shown in (16)- (19). The first problem (15) guarantees that the off-diagonal entries of $\mathcal{G}$ are minimum, the second one (20) makes the on-diagonal elements maximum.

An optimal matrix $U$, that depends on a given $\Gamma$, is obtained by solving the problem (20). By using the method of Lagrange multipliers the following relationships can be written (see appendix A):

$$
\begin{cases}\sum_{r, h}\left(\Gamma_{i r} \Gamma_{i h}-\Gamma_{j r} \Gamma_{j h}\right) U_{i r} U_{j h}=0 & \forall i<j \\ \sum_{r} U_{i r} U_{j r}=\delta_{i j} & \forall i \geq j\end{cases}
$$

that is a system of $n^{2}$ quadratic equations in $n^{2}$ unknown variables, $U_{i j}$. 
3.2 Piezoelectric placement for independent control

An additional optimisation can be performed varying $\Gamma$ through a placement modification of piezoelectric transducers. The targets are to enhance the norm of the matrix $\Gamma$, in order to reach the maximum power flow between the two subsystems, and to diminish the error $\epsilon$ in the approximation of $\mathcal{G}$ with a diagonal matrix, see Eq. (13). It should be noted that such error vanishes as the rows of $\Gamma$ tend to be mutually perpendicular, in this case indeed the matrix $\mathcal{G}$ is absolutely diagonal. To this end, consider the matrix $\Gamma \Gamma^{T}$ can be uniquely decomposed into the sum of two matrices, its diagonal part, $D_{\Gamma \Gamma^{T}}$ and its non-diagonal part, $N_{\Gamma \Gamma^{T}}$. In fact, the diagonal entries of $D_{\Gamma \Gamma^{T}}$ are the squared lengths of the rows of $\Gamma$ and each of them represents the whole power transferred related to the corresponding mechanical dof. Furthermore, the entries below or above the main diagonal of $N_{\Gamma \Gamma^{T}}$ are all the dot products between any two different rows of $\Gamma$ and vanish only if they are perpendicular. Hence, a natural objective function can be introduced as

$$
\mu(\Gamma)=\left\|D_{\Gamma \Gamma^{T}}\right\|-b\left\|N_{\Gamma \Gamma^{T}}\right\|
$$

where $b$ is a proper positive real weight. Now, in accordance with the above, the additional optimisation problem is to find the matrix $\Gamma$ that maximises the objective function (22), that is

$$
\max _{\Gamma \in \mathfrak{S}} \mu(\Gamma)
$$

in which $\mathfrak{S}$ is the set of all possible $\Gamma$. Thus, the goal here is to find a matrix $\Gamma$ whose rows approach to be of maximum length and mutually perpendicular.

\section{Numerical Simulations}

In order to determine characteristics of proposed control, significant numerical cases are considered in this section. At first a numerical analysis is performed on a thin beam with both ends clamped. To further illustrate characteristics of these control a second case is considered involving a rectangular fully clamped plate. Herein, a most important assumption is to consider as piezoelectric transducer two identical thin slice of piezoelectric material bonded symmetrically on both faces of the thin structures and connected in parallel. They are bonded with inverted polarisation directions in order to produce opposite displacement and to induce pure bending. In this way each transducer is associated to one electric dof because of the parallel connection.

Now, recalling that $\mathscr{G}$ is quasi-diagonal and therefore neglecting its off-diagonal entries, it is possible to set $\mathscr{G}_{j k}=g_{j} \delta_{j k}$ and, thus, the equations (10) can be written in the scalar form

$$
\left\{\begin{array}{rl}
\ddot{\eta}_{j}(t)+\omega_{j}^{2} \eta_{j}(t)-\omega_{j} g_{j} \dot{\chi}_{j}(t) & =f_{j}(t) \\
\ddot{\chi}_{j}(t)+\omega_{j} g_{j} \dot{\eta}_{j}(t) & =z_{j}(t)
\end{array}, \quad j=1,2, \ldots n\right.
$$

For each $j$ index, the equations (24) represent a single mode piezoelectric shunting system being the electric forcing term, $z_{j}$, dependent on the generalised electric coordinate, $\chi_{j}$, and its derivatives:

$$
z_{j}=z_{j}\left(\chi_{j}, \dot{\chi}_{j}, \ldots\right), \quad j=1,2, \ldots n
$$

In accordance with Wu's method [11] all the test cases are performed by considering the following relationship for the control action:

$$
z_{j}=-\frac{1}{r_{j}} \dot{\chi}_{j}(t)-\frac{1}{\ell_{j}} \chi_{j}(t), \quad j=1,2, \ldots n
$$

This is a shunt circuit with a resistor, $r_{j}$, and an inductor, $\ell_{j}$, connected in parallel, see Fig. 1.

Computing the optimal values of $r_{j}$ and $\ell_{j}$ for each mode to control, it is possible to assemble the generalised electric forcing vector $\boldsymbol{z}$

$$
z=-\mathscr{R} \dot{\chi}-\mathscr{L} \chi
$$




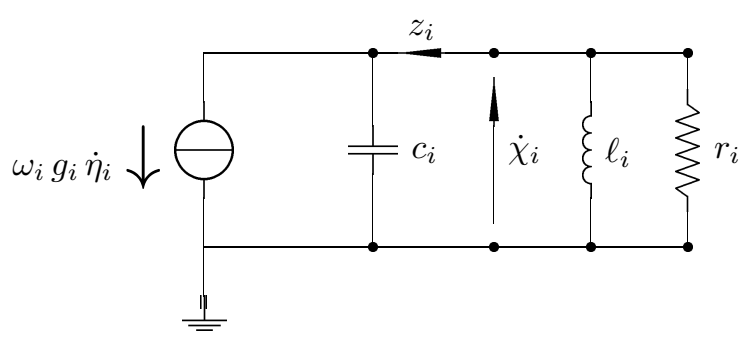

Fig. 1 Equivalent circuit for a virtual passive shunt circuit in parallel configuration.

defining the diagonal matrices $\mathscr{R}_{h k}=\left(1 / r_{h}\right) \delta_{h k}$ and $\mathscr{L}_{h k}=\left(1 / \ell_{h}\right) \delta_{h k}$. Thus, the network constitutive relations get the form

$$
\boldsymbol{\imath}=-\mathcal{N}_{R} \dot{\boldsymbol{\psi}}-\mathcal{N}_{L} \boldsymbol{\psi}
$$

utilising definitions (9) and (11) and setting

$$
\mathcal{N}_{R}=U \mathscr{R} U^{T} \quad \mathcal{N}_{L}=U \mathscr{L} U^{T}
$$

where $\mathcal{N}_{R}$ and $\mathcal{N}_{L}$ are the inductive and the resistive part of the network shunts to the piezoelectric terminals. Because $r_{h}$ and $\ell_{h}$ are all strictly positive and the matrix $U$ is orthogonal, it follows that the network matrices $\mathcal{N}_{R}$ and $\mathcal{N}_{L}$ are symmetric and positive definite. The column vectors of the matrix $U$ can be interpreted as their common eigenvectors, and $\left(1 / r_{h}\right)$ 's and $\left(1 / \ell_{h}\right)$ 's are the corresponding eigenvalues. Besides, to assure their realisation with purely passive components, the network matrices should fulfill other conditions as well as being symmetric and positive definite. A sufficient further condition is the property to be diagonally dominant matrices, see e.g. [17]. Herein, this additional condition has not been considered because the shunt network is implemented with an active feedback control.

\subsection{Finite Element Model}

For all the test cases an accurate estimate for the natural frequencies and corresponding mode shapes, including effects of the piezoelectric transducers under short circuit condition, is obtained by a finite element code ANSYS ${ }^{\circledR}$. The finite element model, here elaborated, is based on the choice of brick elements, soLID226, for the discretisation of piezoelectric patches and of quadrilateral shell elements, SHELL93, for modelling the host structures given that they are designed to model efficiently thin structures.

It is clear that the connection between these two kind of elements is a crucial problem for modelling overall structure. The condition of bonding the piezoelectric material on the host structure is realised in ANSYs by constraint equations, where node displacements of piezoelectric element at the interface with the structure are imposed to make null the relative displacement between the two types of element according to the hypotheses of the Kirchhoff-Love model, as proposed in [18].

The short circuit condition for the piezoelectric elements is modelled by imposing a null voltage on both electrodes of each patch.

To determine the entries of the coupling matrix $\Gamma$, the above finite element model is used.

\subsection{Clamped-clamped beam case study}

The clamped-clamped beam is assumed to be made of aluminium with a rectangular cross section of height $h$ equal to $2.9 \cdot 10^{-3} \mathrm{~m}$ and width $2 \cdot 10^{-2} \mathrm{~m}$ and having length $L$ equal to $0.45 \mathrm{~m}$. Three piezoelectric transducers are bonded on the beam to implement the proposed technique on three mechanical modes. The modes of interest are the lowest because they are prominent in the dynamics of interest. The piezoelectric transducers are assumed to be of ceramic material with properties given in table 1 . The example results given below are for piezoelectric patches of width $b_{p}$ equal to $1.8 \cdot 10^{-2} \mathrm{~m}$ and thickness $h_{p}$ equal to $2.67 \cdot 10^{-4} \mathrm{~m}$. Table 2 displays lengths of the piezoelectric transducers and 


\section{Top view}

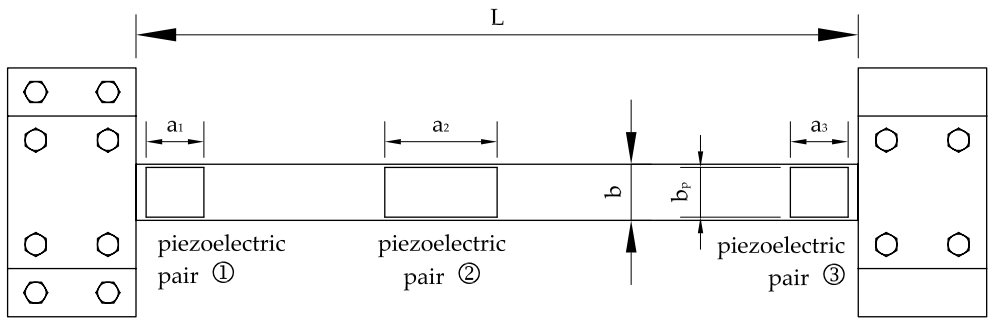

Side view

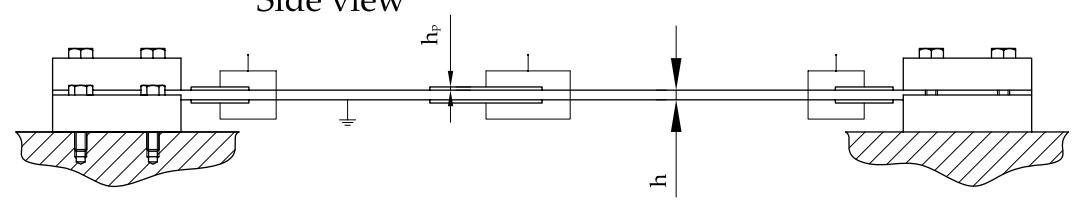

Fig. 2 The beam with three piezoelectric transducers in bending configuration.

Table 1 Material characteristics of the piezoelectric transducers

\begin{tabular}{llll}
\hline & symbol & value & unit \\
\hline Permittivity & $\varepsilon_{33}$ & $3800 \varepsilon_{0}$ & \\
Piezoelectric Strain Constant & $d_{33}$ & $650 \times 10^{-12}$ & $\mathrm{~m} \mathrm{~V}^{-1}$ \\
& $d_{31}$ & $-320 \times 10^{-12}$ & $\mathrm{~m} \mathrm{~V}^{-1}$ \\
Density Mass & $\rho_{p e}$ & 7800 & $\mathrm{~kg} \mathrm{~m}^{-3}$ \\
Young Modulus & $Y_{3}$ & $50 \times 10^{9}$ & $\mathrm{~Pa}$ \\
& $Y_{1}$ & $62 \times 10^{9}$ & $\mathrm{~Pa}$ \\
Poisson's Ratio & $\nu_{p e}$ & 0.31 & \\
\hline
\end{tabular}

their positions defined as the distance of each two-element piezoelectric transducer from one end of the beam. The structure is sketched in Fig. 2.

The modal model of the beam with piezoelectric transducers has the same form presented in Sect. 2. In agreement with classical results [19;20], the expression for the entries of the normalised piezoelectric coupling matrix $\Gamma$ are

$$
\Gamma_{j r}=\frac{d_{31} Y_{1}\left(h+h_{p}\right) b_{p}}{\omega_{j} \sqrt{C_{r}}}\left[\frac{\partial W_{j}}{\partial x_{1}}\right]_{x_{1, i}^{(r)}}^{x_{1, f}^{(r)}}
$$

Herein the notation $\left[f\left(x_{1}\right)\right]_{x_{1, i}}^{x_{1, f}}=f\left(x_{1, f}\right)-f\left(x_{1, i}\right)$ has been used. Let us remember that the piezoelectric material is orthotropic. $d$ is the piezoelectric strain constant, $\varepsilon$ is the permittivity at constant stress. $x_{1, i}^{(r)}$ and $x_{1, f}^{(r)}$ represent respectively the start and end distance of the $r$-th transducer, $\partial W_{j} / \partial x_{1}$ is the mode rotation function of the beam for the $j$-th mechanical mode under short circuit condition and $\omega_{j}$ is the natural frequency associated with it. It should be noted that these entries are also proportional to the average mode curvature of the region covered by the piezoelectric patches. The whole capacitance of the two piezoelectric elements in parallel connection is

$$
C_{r}=2\left(\epsilon_{33}-2 d_{31}^{2} Y_{1}\right) \frac{a_{r} b_{p}}{h_{p}}
$$

The piezoelectric locations are summarised in table 2. Two piezoelectric transducers are placed near to the clamps of the beam and one is located close to, but not exactly, the middle section to sense or excite even the second mode. The lengths are chosen to obtain coupling coefficients with comparable values of bending wavelengths.

In previous sections the assumption that the system possesses negligible mechanical damping is made. At this point the damping effect is included. Introducing this effect and making explicit the 
Table 2 Specifications of the piezoelectric transducers.

\begin{tabular}{lll}
\hline piezo & length $a_{r}(\mathrm{~m})$ & location $x_{1, i}^{(r)}(\mathrm{m})$ \\
\hline 1 & 0.036 & 0.003 \\
2 & 0.07 & 0.155 \\
3 & 0.036 & 0.411 \\
\hline
\end{tabular}

actual electric flux linkage $\phi$, the governing equations for the whole system assume the form

$$
\left\{\begin{aligned}
\ddot{\boldsymbol{\eta}}+D \dot{\boldsymbol{\eta}}+\Omega^{2} \boldsymbol{\eta} & =P \dot{\boldsymbol{\phi}}+\boldsymbol{f} \\
C \ddot{\boldsymbol{\phi}}+\aleph_{p} \dot{\boldsymbol{\phi}} & =-P^{T} \dot{\boldsymbol{\eta}}+\tilde{\boldsymbol{\iota}} \\
\tilde{\boldsymbol{\iota}} & =-\left(N_{R} \dot{\boldsymbol{\phi}}+N_{L} \boldsymbol{\phi}\right)
\end{aligned}\right.
$$

in which $\Omega$ is the diagonal matrix of natural frequencies under the short-circuit condition, $\dot{\boldsymbol{\phi}}=\mathbf{0}$; the matrix $P$ defined as

$$
P=\Omega \Gamma \sqrt{C}
$$

is the piezoelectric coupling matrix; $\boldsymbol{f}$ is the modal mechanical forcing vector. The vector $\boldsymbol{\eta}$ has as elements the modal coordinates. In this case the damping is assumed of the proportional type. This implies that the matrix $D$ is diagonal and can be expressed as

$$
D_{i j}=2 \xi_{j} \omega_{j} \delta_{i j}
$$

where $\xi_{j}$ is the damping ratio of the $j$-th mode assumed equal to $4 \cdot 10^{-3}$ for all modes and $\delta_{i j}$ is the Kronecker symbol. The second equation is the current balance at the electrodes of the piezoelectric elements. The matrix $C$ is a diagonal positive definite matrix of piezoelectric capacitances, whose elements $C_{h} \delta_{h k}$ represent inherent capacitances of piezoelectric patches at blocked modal deflections, $\dot{\boldsymbol{\eta}}=\mathbf{0}$, the matrix $\aleph_{p}$ is a diagonal positive definite matrix, whose entries are $\left(1 / R_{h}\right) \delta_{h k}$ being $R_{h}$ the piezoelectric internal resistances assumed all equal to $10 \mathrm{M} \Omega$; the term $\tilde{\boldsymbol{\iota}}=\sqrt{C} \boldsymbol{\imath}$ is the actual currents flowing through the piezoelectric transducers. The third equation represents the network constitutive relation in which the network matrices are defined as

$$
N_{R}=\sqrt{C} \mathcal{N}_{R} \sqrt{C} \quad N_{L}=\sqrt{C} \mathcal{N}_{L} \sqrt{C}
$$

The system (32) has been modelled with Simulink. The locations used to excite the beam and to compute its response have been chosen to avoid the nodes appearing for low frequency bending modes. In the simulation, the beam is exited by an impulse with a frequency spectrum covering a frequency range from near zero to $f_{\max }$ that is $1200 \mathrm{~Hz}$ at $0.08 \mathrm{~m}$ from the one end of the beam. To model the impulse, a half sine is employed. The peak value, $F_{0}$, is assumed equal to $1 \mathrm{~N}$ and the time duration, $\tau$, is equal to $2.78 \cdot 10^{-4} \mathrm{~s}$ so that $1 / \tau=1.5 f_{\max }$. The time of simulation $T$ is about equal to $10 \mathrm{~s}$, thus the frequency analysis has a frequency resolution $\Delta f$ about equal to $0.1 \mathrm{~Hz}$. The simulation step size is $1 / 12500 \mathrm{~s}$ to avoid computation errors. The output is the velocity at the point $x_{1}$ equal to $0.333 \mathrm{~m}$.

Figure 3 displays the transfer mobilities of the uncontrolled beam with short-circuited piezoelectric transducers and the beam with the optimal passive shunt. The first five modes are considered for the computation of the mobilities and the first three modes are controlled. It is shown that at the first, second and third eigenfrequencies the controlled beam mobility decreases of about $23 \mathrm{~dB}, 22 \mathrm{~dB}$ and $20 \mathrm{~dB}$, respectively. It is still shown that the control does not influence the not controlled forth and fifth modes. Since the same results shown in Fig. 3 are obtained when only the first three controlled modes are considered in the computation, the spillover is negligible.

Figure 4 shows the comparison between impulse response of the controlled and uncontrolled beam. The controlled response decreases faster than the uncontrolled one. In fact, at $0.15 \mathrm{~s}$ the controlled response reduces of $99.8 \%$, instead of the uncontrolled one that decreases of $98 \%$ at $1 \mathrm{~s}$. 


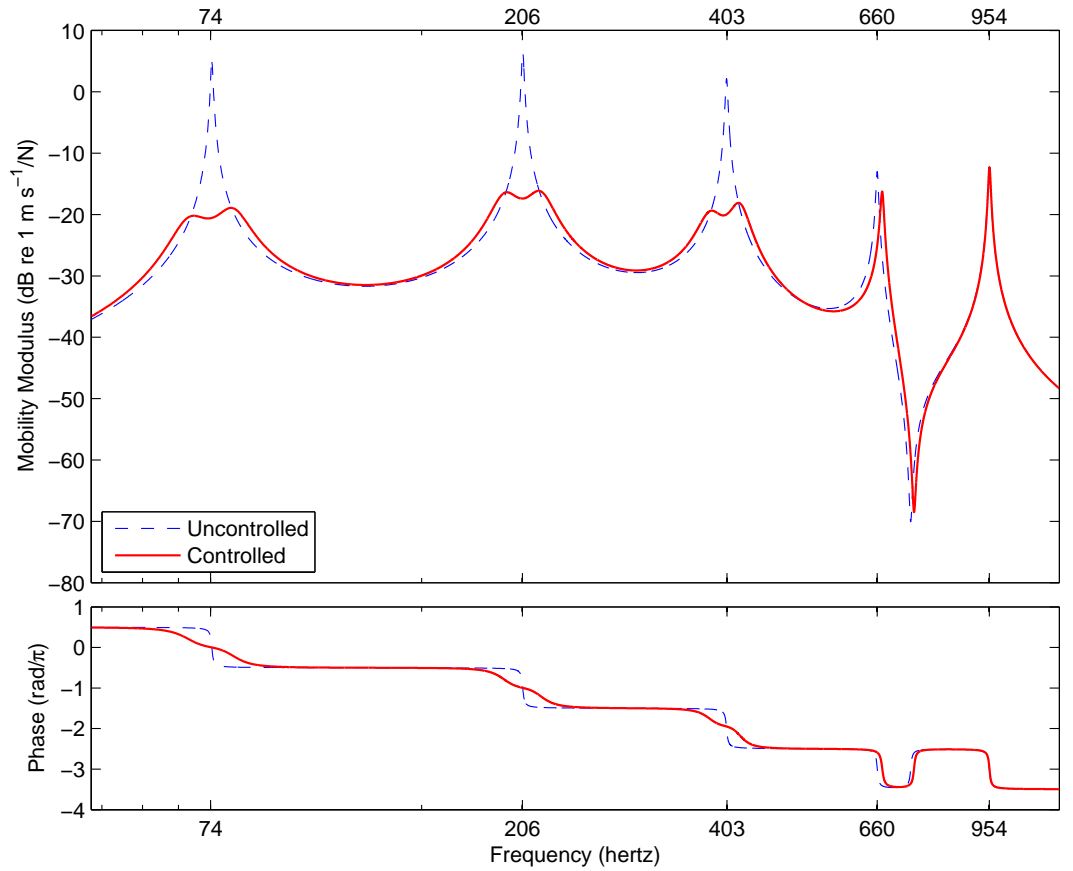

Fig. 3 Comparison between mobility of the controlled and uncontrolled beam: three modes considered.

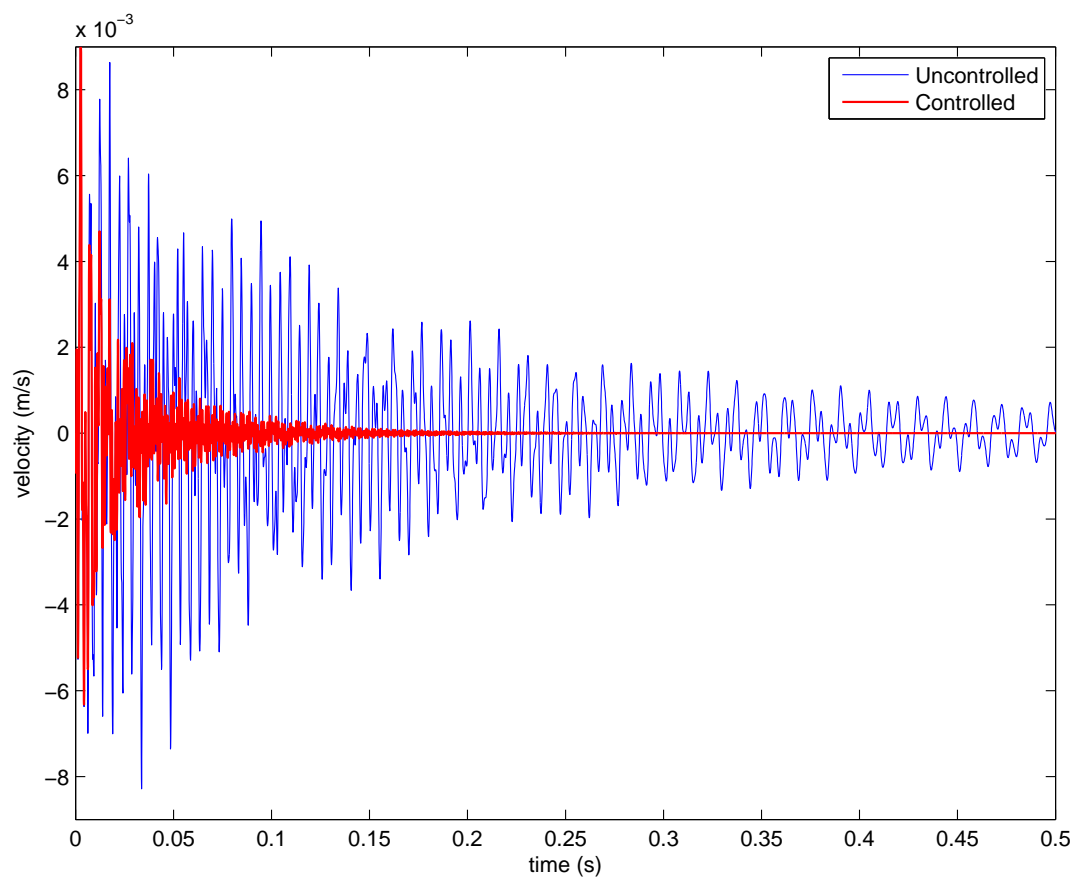

Fig. 4 Comparison between impulse response of the controlled and uncontrolled beam. 


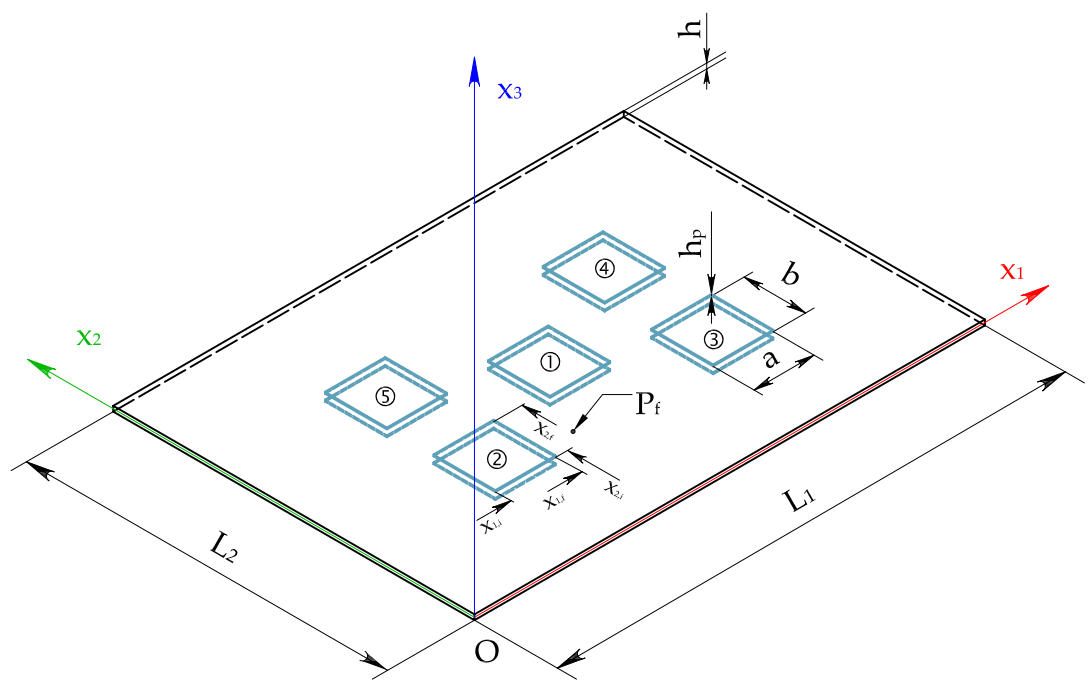

Fig. 5 The plate with five piezoelectric transducers in bending configuration.

Table 3 Geometric and material characteristics of the plate.

\begin{tabular}{llll}
\hline & symbol & value & unit \\
\hline Length & $L_{1}$ & 0.297 & $\mathrm{~m}$ \\
Width & $L_{2}$ & 0.210 & $\mathrm{~m}$ \\
Thickness & $h$ & 0.001 & $\mathrm{~m}$ \\
Density mass & $\rho$ & 2700 & $\mathrm{~kg} \mathrm{~m}^{-3}$ \\
Young modulus & $Y$ & $69 \times 10^{9}$ & $\mathrm{~Pa}$ \\
Poisson's ratio & $\nu$ & 0.33 & \\
\hline
\end{tabular}

\subsection{Fully clamped plate case study}

The plate investigated in this case study is a rectangular fully clamped aluminium plate with multiple piezoelectric transducers according to Fig. 5. In order to control five mechanical modes, the proposed control uses five piezoelectric transducers in bending configuration. Let the five transducers be identical. The model used below is summarised in appendix B. The domain $\mathscr{A}$ denotes the region occupied by the plate and $\mathscr{A}_{k}$ is the region occupied by the $k$-th transducer. Tables 3 and 1 report the material and geometric characteristics of the plate and of the piezoelectric elements.

This section presents an example that illustrates the optimisation problem of the transducer allocation on the plate using the procedure outlined in Sect. 3.2 based on the index (22). To set up the problem, one piezoelectric transducer out of five, $\mathscr{A}_{1}$, is placed at the centre of the plate a priori. The other four piezoelectric transducers are located to form a symmetric pattern as shown in Fig. 5. Lengths, $a$, and widths, $b$, of the piezoelectric laminae are fixed equal and comparable with wavelengths of the modes of interest. Thus, the edges of the piezoelectric elements are $3.6 \cdot 10^{-2} \mathrm{~m}$, whilst the thickness $h_{p}$ is equal to $2.67 \cdot 10^{-4} \mathrm{~m}$. These choices allow to make a parameterization of the transducer arrangement by means of two scalar parameters $\left[\alpha_{1}, \alpha_{2}\right]$ which represent the coordinates of left lower corner, $\left[x_{1, i}^{(2)}, x_{2, i}^{(2)}\right]$, of the piezoelectric pair $\mathscr{A}_{2}$. Holding their symmetry the positions of piezoelectric transducers from $\mathscr{A}_{2}$ to $\mathscr{A}_{5}$ are dependent only on the position of $\mathscr{A}_{2}$. Thus, the goal in this example is to find a set of values $\left[\tilde{\alpha}_{1}, \tilde{\alpha}_{2}\right]$ that maximise the index $(22)$, that is

$$
\mu(\Gamma)=\left\|D_{\Gamma \Gamma^{T}}\right\|-\frac{1}{2}\left\|N_{\Gamma \Gamma^{T}}\right\|
$$

as parameters $\left[\alpha_{1}, \alpha_{2}\right]$, subject to the constraint to avoid overlap between transducers, vary in a quarter of the plate. This optimisation problem is iterative and requires the computation of the whole finite element model at each step, thus it is very expensive. For this reason, a roughly analysis is previously performed using mode shapes and natural frequencies of the plate without transducers. This analysis 


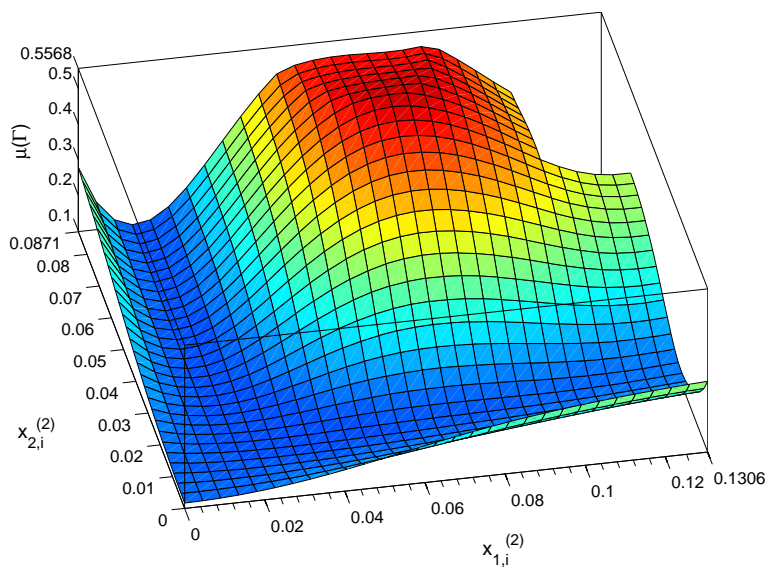

Fig. 6 Piezoelectric placement objective function $\mu(\Gamma)$ for a quarter of plate.

permits to make faster the following finite element analysis using as initial guess for the parameter vector $\left[\alpha_{1}, \alpha_{2}\right]$ the optimal parameter vector obtained in this preceding step, see figure 6 . Thus, the starting guess is initialised to $[0.0622,0.0550]$ and the optimal parameter vector obtained with the finite element model is

$$
\left[\tilde{\alpha}_{1}, \tilde{\alpha}_{2}\right]=[0.0676,0.0554]
$$

which corresponds to the position of the piezoelectric elements represented in Fig. 5. Note that the finite element analysis yields little different result from the preliminary one, so it could be safely ignored.

In this case the piezoelectric coupling matrix relative to the generalised electric coordinates $\chi_{k}(t)$ becomes

$$
\mathscr{G}=\left(\begin{array}{rrrrr}
0.250 & 0 & 0 & 9.75 \cdot 10^{-4} & 0 \\
0 & 0.302 & 0 & 0 & 0 \\
0 & 0 & 0.297 & 0 & 0 \\
7.84 \cdot 10^{-4} & 0 & 0 & 0.323 & 0 \\
0 & 0 & 0 & 0 & 0.396
\end{array}\right)
$$

Note that the second step of optimisation introduced in Sect. 3.2 allows to obtain a coupling matrix $\Gamma$ with all rows mutually perpendicular. Only the angle $\vartheta_{14}$ between the first row and the fourth row of $\Gamma$ is a slightly different, that is $0.498 \pi$.

To validate damping performances of the proposed control, a Simulink model with the same layout as the beam is used. In detail, the plate is excited by an impulse with a frequency spectrum over a frequency range from near zero to $f_{\max }$ that is $1000 \mathrm{~Hz}$. The mobility is considered between the transversal force applied at the point $P_{f}$ shown in Fig. 5, and the transversal velocity calculated at the same point. The position of the point $P_{f}$ is chosen to excite and observe all modes in the frequency range of interest by avoiding nodal lines as more as possible. The impulse has a peak value $F_{0}$ of $1 \mathrm{~N}$ and a time duration $\tau$ of $3.33 \cdot 10^{-4}$ s so that $1 / \tau=1.5 f_{\max }$. The time of simulation $T$ is about equal to $10 \mathrm{~s}$. The simulation step size is $1 / 12500 \mathrm{~s}$. To take into account the spillover problem the system model consists of ten mechanical modes instead of five. Figure 7 displays the point mobilities of the uncontrolled plate with short-circuited piezoelectric elements and the controlled plate with the optimal shunting network. The proposed control is equally effective on all modes acting simultaneously on five picks. Figure 8 displays the comparison between impulse response of the controlled and uncontrolled plate. The controlled response reduces of $97.4 \%$ at $0.15 \mathrm{~s}$, while the uncontrolled one has the same decrement at $0.5 \mathrm{~s}$.

Several authors extended the technique of the single shunt to damp multiple mechanical modes [12; 4; 21]. They shunt a single piezoelectric element with a circuit including several resistors, capacitors, and inductors, to obtain a multi-resonant behaviour. In Fig. 9 the comparison between the result given by the technique proposed in this paper and that obtained by the multi-resonant current flowing circuits proposed in [21] is shown (see appendix C). The main feature of the proposed technique is to exploit the coupling of the different piezoelectric transducers in an optimal way, by the maximisation 


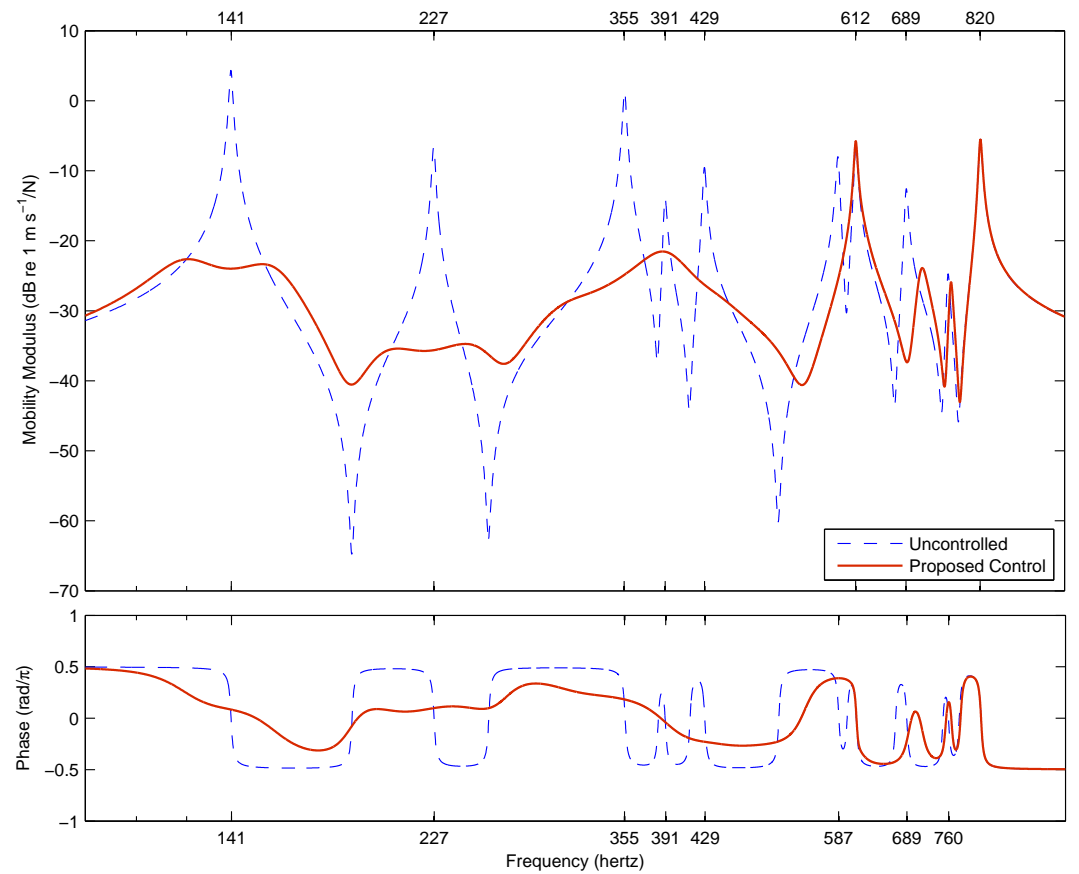

Fig. 7 Comparison between mobility of the controlled and uncontrolled beam: the first five modes considered.

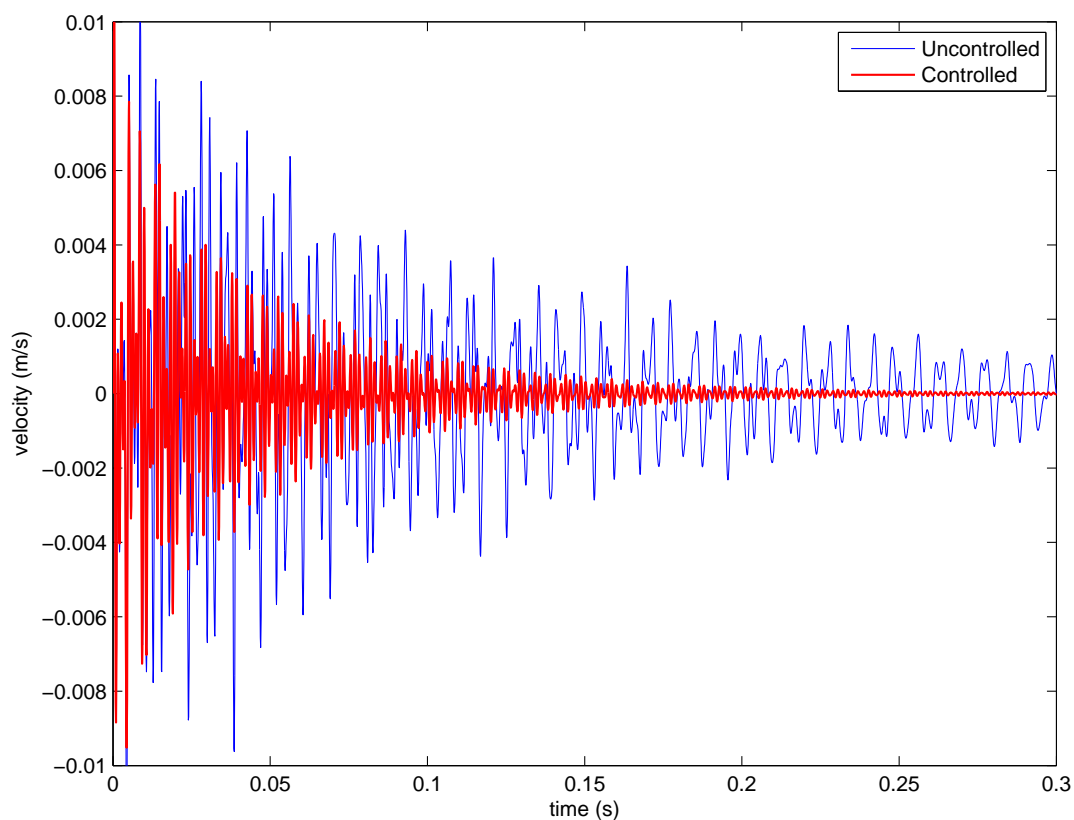

Fig. 8 Comparison between impulse response of the controlled and uncontrolled beam.

of the transfer power and improvement of the energy exchange efficiency between the mechanical and electrical system. Furthermore, the proposed approach does not employ additional capacitances which reduce the efficiency of electromechanical coupling. The computational solution obtained by the proposed technique allows to reach a maximum reduction in the magnitude of mobility of about 25 $\mathrm{dB}$, instead of about $15 \mathrm{~dB}$ of the result of the approach proposed in [21]. 


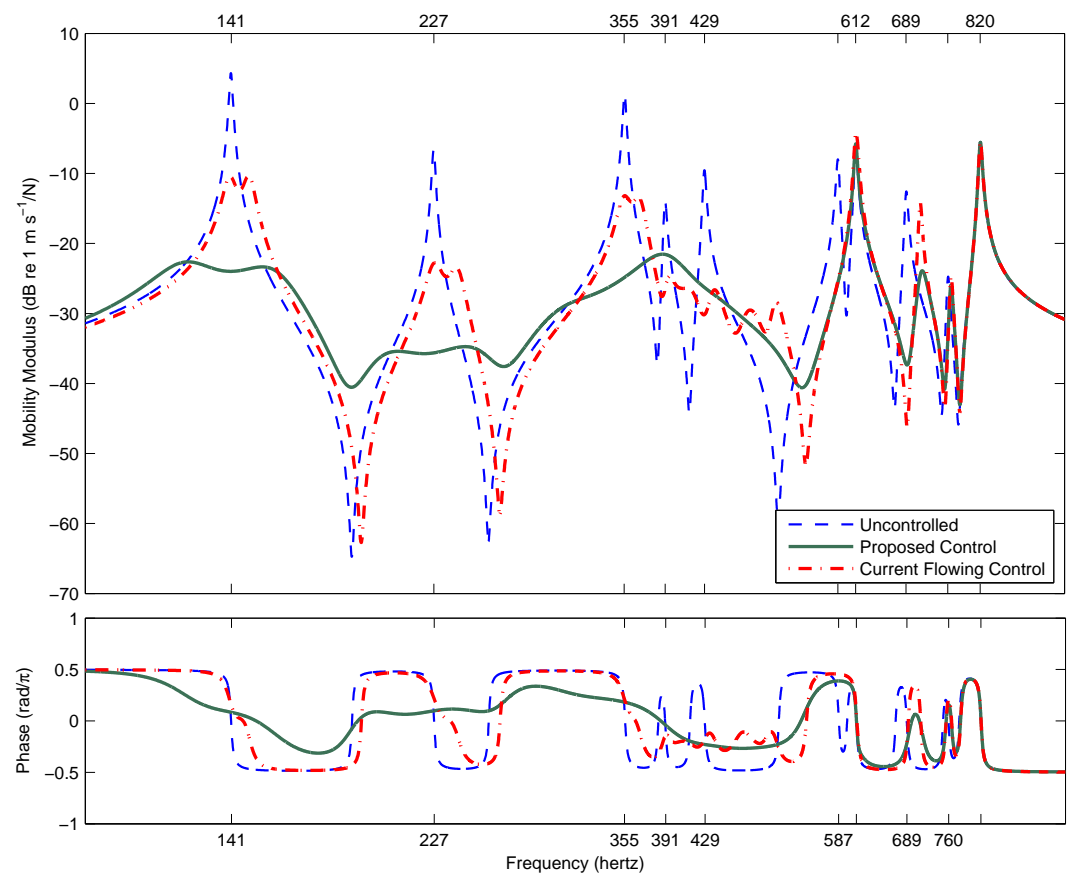

Fig. 9 Mobility of the uncontrolled plate and the comparison with the proposed control and the current flowing control on the first five modes.

\section{Laboratory Experiments}

To test the effectiveness of proposed controls, an experimental case is considered in this section. The analysis is performed on a thin beam with both ends clamped, the same described in section 4. First, a system parameter identification is accomplished by means of system frequency response functions (FRF's). Then, the results of proposed technique are presented and are compared with those obtained with numerical models. Particularly, the shunting network is implemented by means of an active feedback control to avoid actualisation issues as the requirement of large inductor values.

\subsection{Experimental set up}

The test system is a uniform aluminium beam with rectangular cross section and experimentally clamped boundary conditions at both ends. Great attention has been devoted to fix end points of the beam to ground, in order to ensure that the whole assembly gives repeatable results. One of the tackled problems is the change in stiffness due to axial load caused by environment temperature variations. To avoid this problem the axial displacement at one end is allowed. Three pairs of piezoelectric ceramic rectangular patches, connected in parallel, are bonded symmetrically to either side of the beam surface. They are used as actuator to provide disturbing force, as sensor and also as shunting layer. The experimental set up used to measure the FRF's of interest is a typical layout for measurement or for the active feedback control. The measurement chain consists in:

1. a $P c$ based data acquisition and arbitrary waveform generation system;

2. a piezoelectric transducer used as actuator to introduce external disturbance or driving excitation;

3. a power amplifier to drive with voltage or current piezoelectric patches;

4. two piezoelectric transducers used as shunting layer for the active feedback control;

5. sensors to measure the various responses of interest: accelerometers, a laser vibrometer and piezoelectric transducers. 

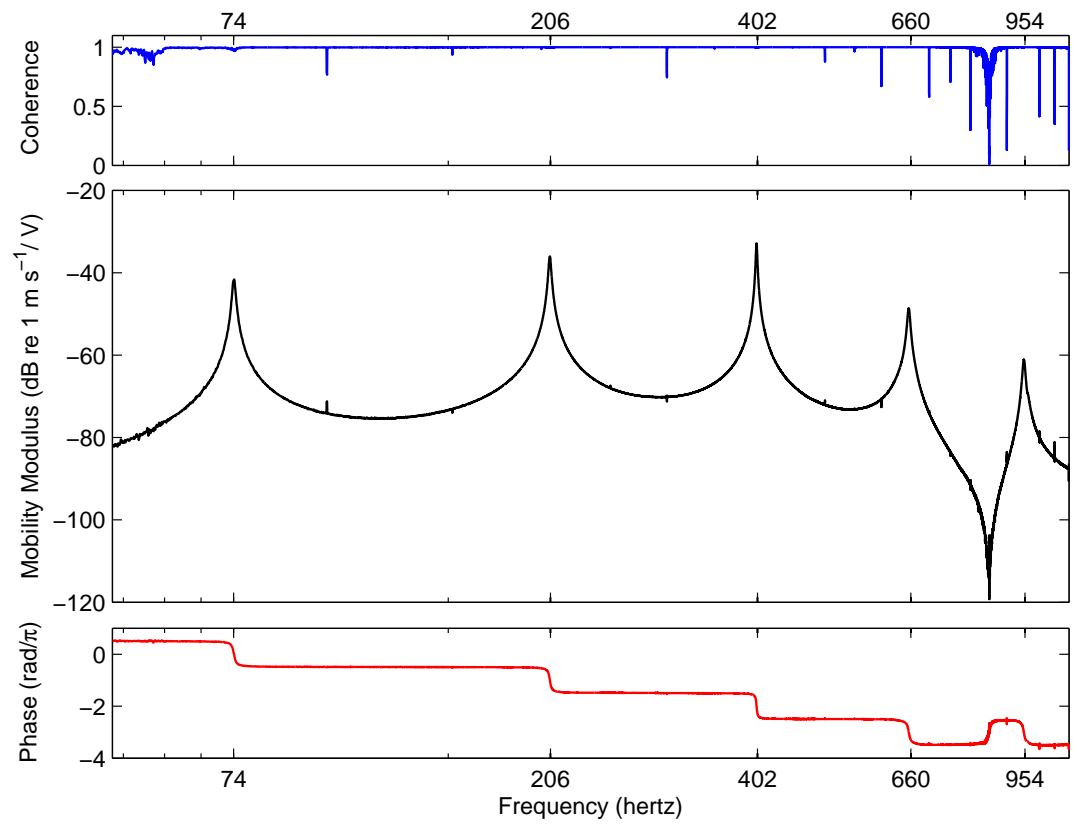

Fig. 10 Mobility function of the beam.

Table 4 Resonance frequencies of the beam.

\begin{tabular}{llll}
\hline & experimental $(\mathrm{Hz})$ & numerical $(\mathrm{Hz})$ & discrepancy $(\%)$ \\
\hline mode 1 & 74.3 & 74.6 & 0.4 \\
mode 2 & 206.2 & 209.6 & 1.6 \\
mode 3 & 402.6 & 405.6 & 0.7 \\
mode 4 & 660.2 & 662.4 & 0.7 \\
\hline
\end{tabular}

\subsection{System Identification}

To extract modal parameters, that is resonance frequencies and related damping ratios, the beam is excited by the piezoelectric pair 1 with a random band-limited signal so as to excite the bending modes of interest with a frequency spectrum covering a range from $50 \mathrm{~Hz}$ to $1000 \mathrm{~Hz}$. A power amplifier to drive the piezoelectric actuator with voltage is used. As sensor a laser vibrometer is used. The time history signals are $10 \mathrm{~s}$ long. Figure 10 shows the frequency response function obtained. The estimated eigenfrequencies are summarised in table 4 in which there are even the resonance frequencies obtained by the finite element analysis for comparing experimental and numerical results.

The piezoelectric inherent capacitances, are measured by a series $L C$ circuit. The quantity $C$ of the circuit is the piezoelectric inherent capacitance, the inductance $L$ is a variable floating inductor made by active components. The $L C$ circuit is excited by a voltage source and it is measured the voltage on the piezoelectric terminals. Thus, the electric resonance frequency, $\omega_{L C}$, can be obtained by analysing the frequency spectrum. By tuning the inductance, the frequency $\omega_{L C}$ is set in a frequency range where the mechanical effect can be neglected. For this value of electric eigenfrequency the corresponding inductance is measured. Therefore, the piezoelectric capacitance is calculated by the following relationship $C=1 /\left(L \omega_{L C}^{2}\right)$. The results are

$$
C_{1}=111.3 \mathrm{nF}, \quad C_{2}=216.5 \mathrm{nF} \quad \text { and } \quad C_{3}=104.8 \mathrm{nF}
$$

\subsubsection{Coupling coefficients}

The identification procedure used to measure the coupling parameters, that are the entries of the matrix $\Gamma$, exploits the classical results based on the inductive-resistive single resonant piezoelectric 
Table 5 Normalised piezoelectric coupling coefficients.

\begin{tabular}{llll}
\hline & piezo 1 & piezo 2 & piezo 3 \\
\hline mode 1 & -0.0944 & 0.114 & -0.105 \\
mode 2 & 0.0821 & -0.0868 & -0.0915 \\
mode 3 & -0.0619 & -0.0801 & -0.0722 \\
mode 4 & -0.0533 & -0.141 & 0.0533 \\
\hline
\end{tabular}

Table 6 Numerical normalised piezoelectric coupling coefficients.

\begin{tabular}{lllllll}
\hline & piezo 1 & $\Delta 1(\%)$ & piezo 2 & $\Delta 2(\%)$ & piezo 3 & $\Delta 3(\%)$ \\
\hline mode 1 & -0.1106 & 14 & 0.1327 & 9 & -0.1105 & 5 \\
mode 2 & 0.0965 & 14 & -0.0973 & 7 & -0.0987 & 9 \\
mode 3 & -0.0802 & 15 & -0.1025 & 12 & -0.0828 & 6 \\
mode 4 & -0.0637 & 21 & -0.1417 & 1 & 0.0610 & 2 \\
\hline
\end{tabular}

shunt circuit and the fixed point theory. For further details the reader is referred to the paper [22]. The beam is excited through one piezoelectric transducer by a voltage source using a chirp of amplitude $1 \mathrm{~V}$ which rises linearly in frequency nearly to the mechanical eigenfrequency of interest for a time of $10 \mathrm{~s}$. A second piezoelectric transducer is used as sensor, to obtain the frequency response function of the system. The last piezoelectric transducer is shunted with a series $R L$ tuned circuit where the inductor is a variable device op-amp based. According with the fixed point theory, the coupling parameters are calculated using the following relation

$$
\Gamma_{j r}=\sqrt{-\beta_{j r}^{2}+\sqrt{\beta_{j r}^{4}+\frac{\left(\omega_{T}^{2}-\omega_{S}^{2}\right)^{2}}{\omega_{j}^{4}}}}
$$

where $\beta_{j r}$ is the ratio of the electric resonance of the $R L C$ shunt circuit on the $r$-th piezoelectric transducer and the mechanical eigenfrequency $\omega_{j}$. The frequencies $\omega_{S}$ and $\omega_{T}$ are the fixed points related to several values of resistance as depicted in Fig. 11. The inductance is tuned to have a ratio $\beta_{j r}$ equal to 1 . The obtained results are shown in table 5 . In table 6 are shown the coupling coefficients obtained via a finite element model and the comparison between direct measurement and numerical prediction.

\subsection{Control Validation}

To test the proposed control, laboratory experiments are carried out in real time. In particular, the MathWorks xPC Target is used to create a real-time controller using a standard PC. MATLAB and SimUlinK are used to create a model of the shunting network (see Fig. 12). It should be noted that model size, complexity, and target PC hardware affect minimal sample time of execution. The model employed (Fig. 12) can run with a sample time as fast as $33.3 \mu \mathrm{s}$, i.e. $30 \mathrm{kHz}$.

The shunt network system is realised through an active feedback control considering as measurement signal the voltage on each piezoelectric transducer and as control signal the current flowing through the same piezoelectric transducer in order to mimic the network behaviour. The chosen set up is primarily due to the following aspects: the easiness to measure high voltage and to supply current with required accuracy on a piezoelectric transducer, as well as the minor influence of the hysteretic phenomena of the piezoelectric transducers driven by current source.

To check the validity of control, a dual channel FFT analysis is performed to calculate a $F R F$ of the beam. The disturbance actuator is one piezoelectric transducer driven by a linear chirp waveform with a frequency range from $50 \mathrm{~Hz}$ to $800 \mathrm{~Hz}$ and an amplitude equal to $3 \mathrm{~V}$. An accelerometer is used to measure the response of the system and, therefore, to calculate the $F R F$. The other two piezoelectric transducers are employed to control the beam vibrations. The acquisition time is $10 \mathrm{~s}$, hence the frequency analysis has a frequency resolution $\Delta f=0.1 \mathrm{~Hz}$. The figures $13-15$ show the transfer inertance and the comparison between the system response without control and with the 


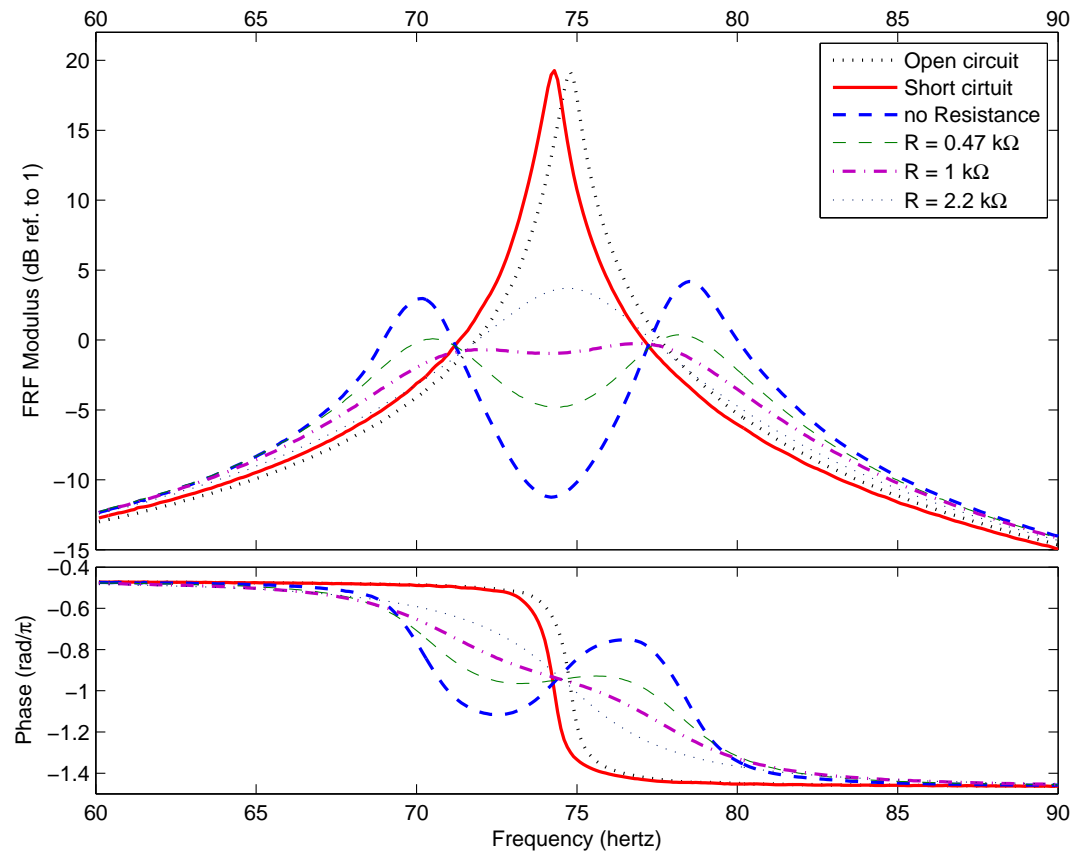

Fig. $11 F R F$ of the beam with shunt circuit on the central piezoelectric pair close to the first beam mode.

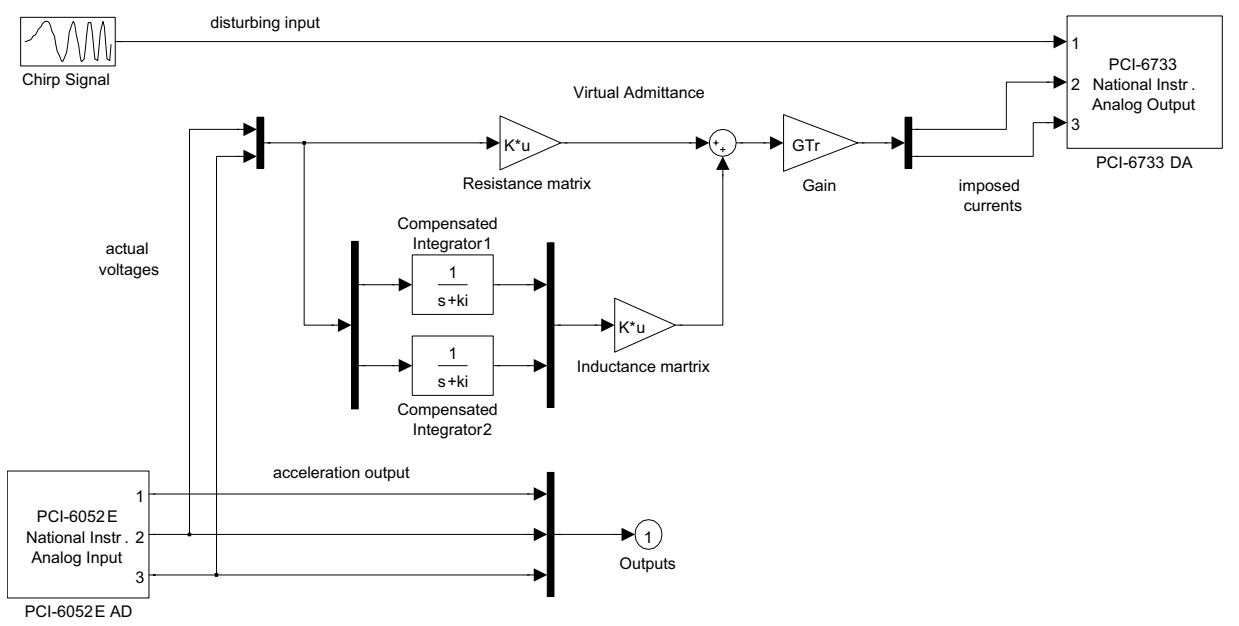

Fig. 12 Simulink diagram for the real-time application.

control applied on two modes. It is shown as the passive network system acts over different pair of modes attesting to be equally able to accomplish the purpose of reducing vibrations.

\section{Conclusions}

The main result of this work is an extension of the known piezoelectric shunt damping techniques. We illustrated a method for controlling $n$ structural modes by $n$ piezoelectric transducers shunted with a multiterminal electric network system. Differing from existing approaches which consider sev- 

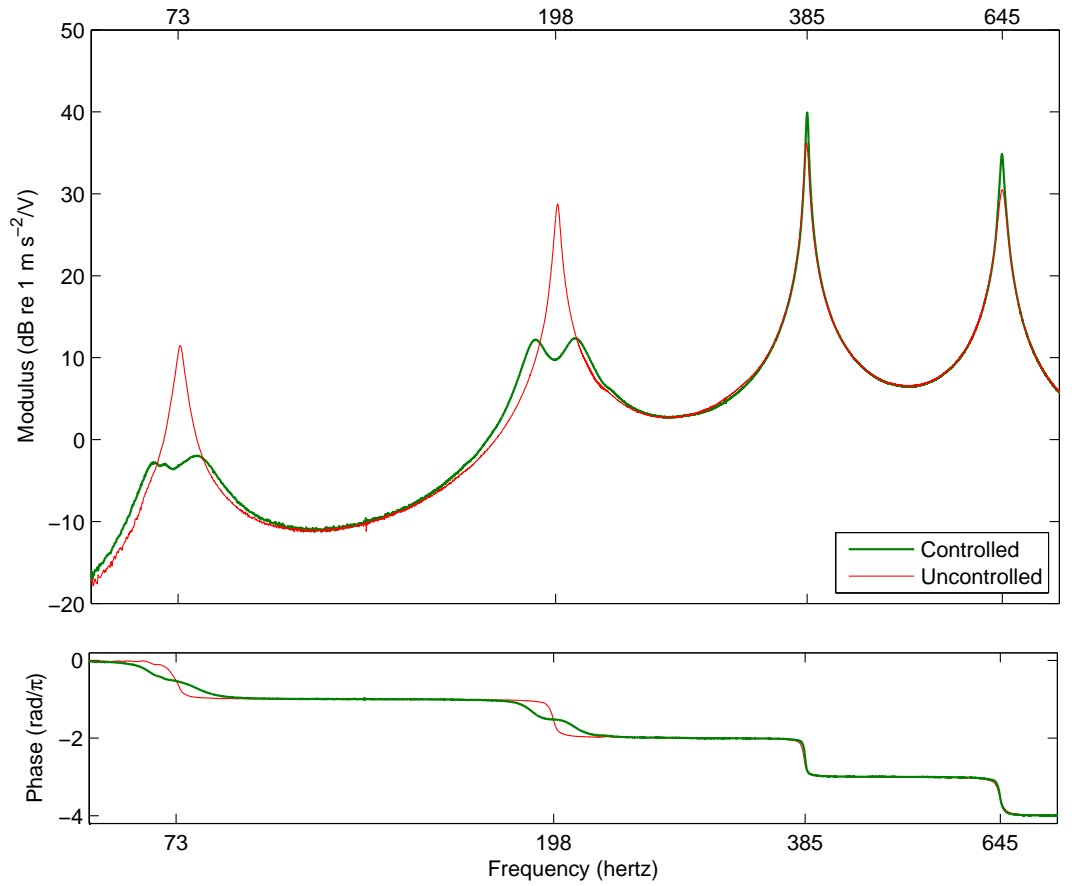

Fig. 13 Inertance function of the beam and comparison with proposed control for the first and the second modes.
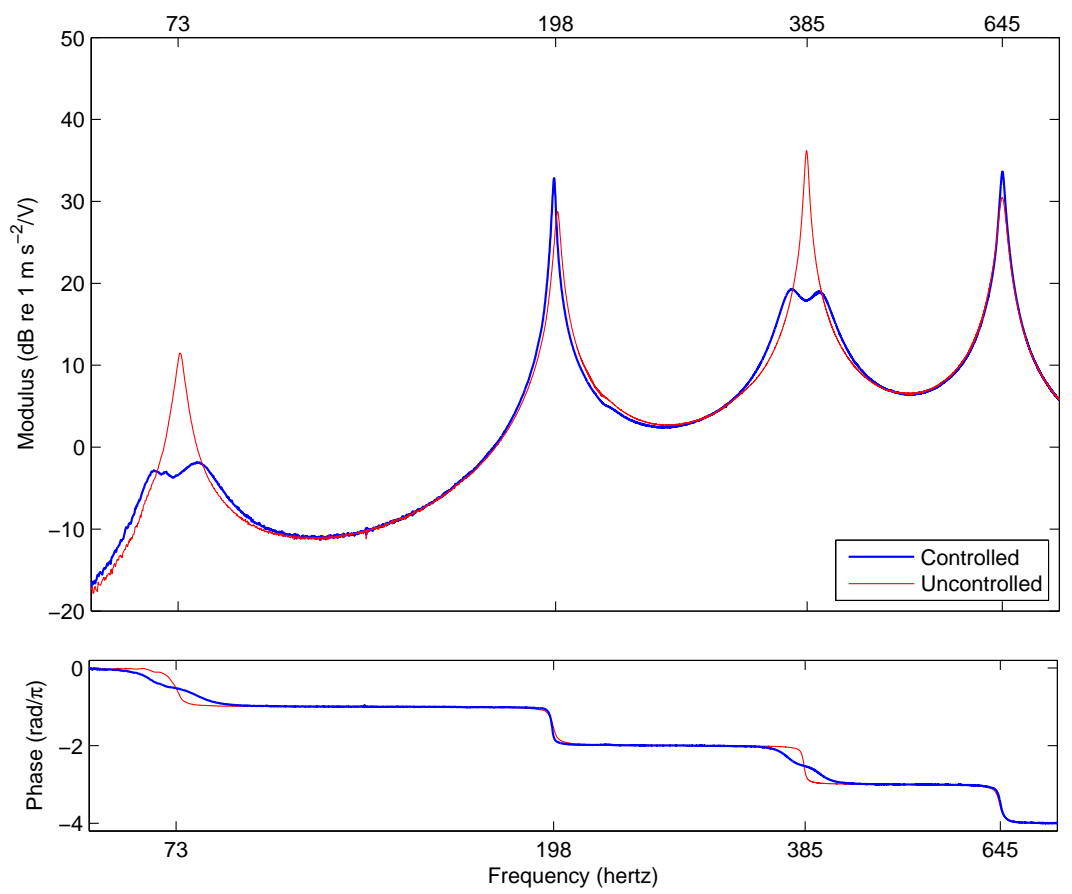

Fig. 14 Inertance function of the beam and comparison with proposed control for the first and the third modes. 

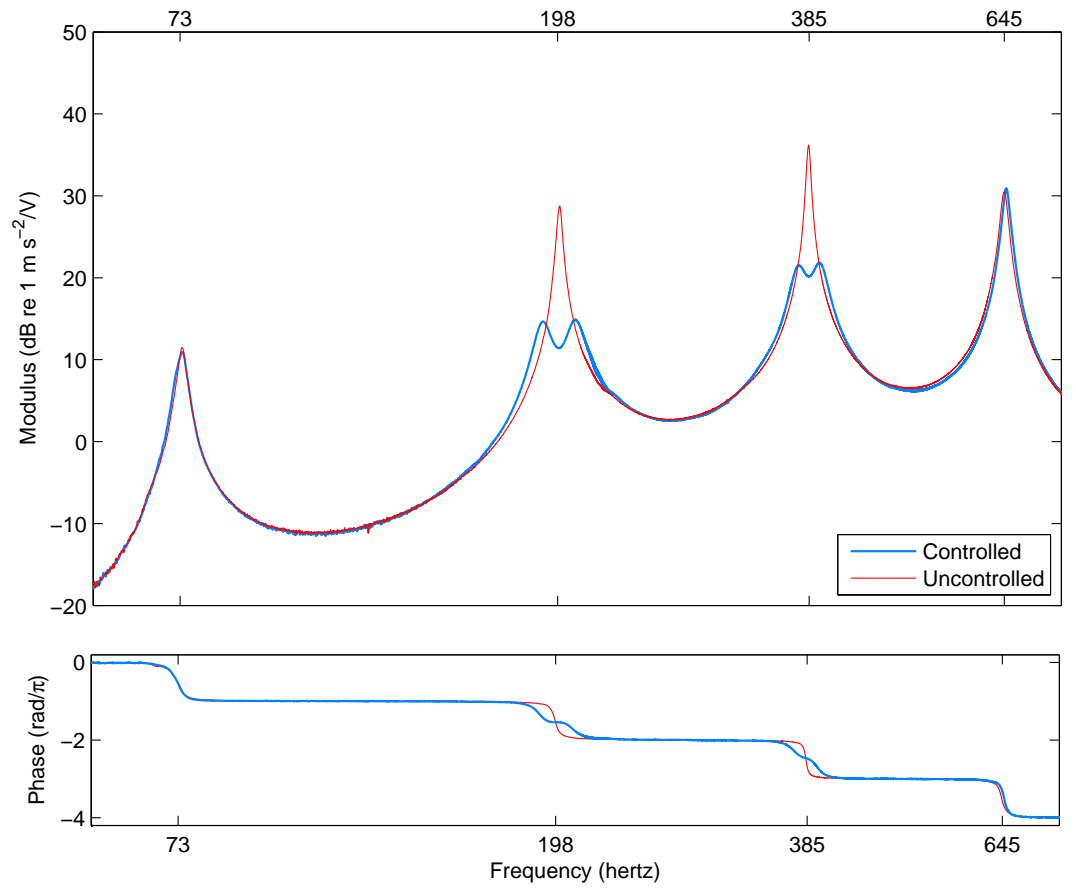

Fig. 15 Inertance function of the beam and comparison with proposed control for the second and the third modes.

eral separately shunts, our shunting network interconnects the piezoelectric transducers for a spatial, simultaneous control of different mechanical modes. The shunting network forms, with the $n$ inherent piezoelectric capacitances at blocked modal deflections, an $n$ dof's electric system having the following modal properties: the same natural frequencies of the set of mechanical modes to be controlled, optimal damping ratios to absorb the mechanical energy.

Two optimisation problems were shown. By introducing a proper transformation of electric coordinates, a one to one correspondence between the modal mechanical and new electric dof is approximately attained. Further, the distribution of the piezoelectric transducers is improved to maximise the damping performance.

Two numerical cases, a double clumped beam and a fully clamped plate, was developed to validate the technique. The numerical simulations, obtained with the aid of Simulink, show that the damping performances are sensibly increased with respect to existing techniques for multimodal damping. This is mainly due two reasons: the effective optimal use of all the transducers for all the modes, the avoidance of the usage of additional external capacitors, which decrease the electromechanical couplings. Besides, an experimental test case was accomplished: double clamped beam. In experimental implementations, the required shunting network was obtained by an active feedback control, using multiple voltagecontrolled current sources.

The obtained shunt damping network is passive because the network matrices are positive definite so the present technique can be classified as a virtual passive damping [23] and has the main advantage with respect to purely active approaches to be unconditionally stable.

\section{Appendix A: Optimisation problem}

The optimisation problem (20) can be solved by using the method of Lagrange multipliers. To this end, let $\vartheta(U)$ the objective function

$$
\vartheta(U)=D_{\Gamma U} \cdot D_{\Gamma U}
$$

subject to the orthogonal constraint

$$
U U^{T}-I=O
$$


where $I$ is the identity matrix and $O$ the zero matrix both of size $n$. Now, define the Lagrangian, $\Lambda$, as

$$
\Lambda(U, S)=D_{\Gamma U} \cdot D_{\Gamma U}-\left(U U^{T}-I\right) \cdot S
$$

where $S$ is a symmetric matrix of undetermined multipliers. Setting the partial derivatives of $\Lambda$ respect to $U$ and $S$ equal to zero, it is possible to write the system of equations

$$
\left\{\begin{array}{l}
S U=\Gamma^{T} D_{\Gamma U} \\
U U^{T}-I=O
\end{array}\right.
$$

which solved yields the stationary values for the objective function (A-1). In detail, the Lagrange multiplier matrix $S$ can be expressed as

$$
S=\Gamma^{T} D_{\Gamma U} U^{T}
$$

Since the matrix $S$ must be symmetric

$$
S=\Gamma^{T} D_{\Gamma U} U^{T}=U D_{\Gamma U} \Gamma=S^{T}
$$

the first equation of the (A-4) becomes

$$
(\Gamma U)^{T} D_{\Gamma U}=D_{\Gamma U} \Gamma U
$$

Finally, taking only the significant equations of the (A-7) and (A-2), the equation set that solved the problem (20) consists of the following equations

$$
\begin{cases}\sum_{r, h}\left(\Gamma_{i r} \Gamma_{i h}-\Gamma_{j r} \Gamma_{j h}\right) U_{i r} U_{j h}=0 & \forall i<j \\ \sum_{r} U_{i r} U_{j r}=\delta_{i j} & \forall i \geq j\end{cases}
$$

There is a geometric interpretation of this system. Each equation determines a quadric in $n^{2}$-dimensional space and hence, the solution set is the intersection of these quadrics.

\section{Appendix B: Modal analysis of the plate} follows

The governing equation of the plate with several piezoelectric transducers can be rewritten as

$$
\left\{\begin{array}{l}
\tilde{B} \nabla^{4} w+\tilde{m} \frac{\partial^{2} w}{\partial t^{2}}=p+\sum_{r=1}^{n_{p}} \frac{K_{m e}\left(h+h_{p}\right)}{\sqrt{C_{r}}} \nabla^{2} \wp_{r} \frac{d \psi_{r}}{d t} \\
\mathcal{Q}_{r}=\frac{d \psi_{r}}{d t}+\int_{\mathscr{A}_{r}} \frac{K_{m e}\left(h+h_{p}\right)}{\sqrt{C_{r}}}\left(\frac{\partial^{2} w}{\partial x_{1}^{2}}+\frac{\partial^{2} w}{\partial x_{2}^{2}}\right) d \mathscr{A}_{r}, \quad r=1,2, \ldots n_{p}
\end{array}\right.
$$

Let us remember that the plate material is isotropic and the piezoelectric material is orthotropic. The subscript $p$ is related to piezoelectric properties. The material constants $K_{m m}, K_{m e}$ and $K_{e e}$ are given by

$$
\begin{aligned}
K_{m m} & =\frac{Y_{1}}{1-\nu_{12}^{2}} \\
K_{m e} & =d_{31} \frac{Y_{1}}{1-\nu_{12}} \\
K_{e e} & =\left(\epsilon_{33}-2 d_{31}{ }^{2} \frac{Y_{1}}{1-\nu_{12}}\right)
\end{aligned}
$$


Table 7 Parameters of the five current flowing circuits identical used for comparison.

\begin{tabular}{lllllll}
\hline & & I branch & II branch & III branch & IV branch & V branch \\
\hline$L_{i}$ & $\mathrm{H}$ & 89.61 & 33.98 & 13.97 & 11.14 & 8.84 \\
$R_{i}$ & $\Omega$ & 4500 & 3500 & 1700 & 1500 & 1500 \\
$K_{i}$ & $\mathrm{nF}$ & 14.6 & 14.6 & 14.6 & 14.6 & 14.6 \\
\hline
\end{tabular}

$\mathscr{A}_{r}$ is the area occupied by each piezoelectric pair, $C_{r}=2\left(K_{e e} / h_{p}\right) \mathscr{A}_{r}$ is the overall inherent capacitance of the $r$-th piezoelectric pair, the total bending stiffness and the total mass per unit of surface are

$$
\begin{aligned}
\tilde{B} & =B+\sum_{r=1}^{n_{p}} K_{m m}\left(\frac{h^{2} h_{p}}{2}+h h_{p}^{2}+\frac{2}{3} h_{p}^{3}\right) \wp_{r} \\
\tilde{m} & =\rho h+2 \sum_{r=1}^{n_{p}} \rho_{p} h_{p} \wp_{r}
\end{aligned}
$$

In order to consider the eigenvalue problem (B-1), the boundary conditions of fully clamped edges for the transverse displacement, $w$, and the short circuit condition for normalised voltage, $\dot{\psi}_{r}$, are assumed.

The mode model of the smart plate can be given in the form of $\mathrm{Eq}(8)$ where

$$
\Gamma_{i r}=\frac{K_{m e}\left(h+h_{p}\right)}{\omega_{i} \sqrt{C_{r}}}\left(\int_{x_{2, i}^{(r)}}^{x_{2, f}^{(r)}}\left[\frac{\partial W_{i}}{\partial x_{1}}\right]_{x_{1, i}^{(r)}}^{x_{1, f}^{(r)}} d x_{2}+\int_{x_{1, i}^{(r)}}^{x_{1, f}^{(r)}}\left[\frac{\partial W_{i}}{\partial x_{2}}\right]_{x_{2, i}^{(r)}}^{x_{2, f}^{(r)}} d x_{1}\right)
$$

and

$$
f_{i}=\int_{\mathscr{A}} p\left(x_{1}, x_{2}, t\right) W_{i} d \mathscr{A} \quad i=1, \ldots n_{m}
$$

\section{Appendix C: The current flowing shunt circuit}

The current flowing shunt circuit proposed in [21] seems the most efficient thanks to the modular circuital pattern and the reduced number of elements. The shunt has as many parallel branches as the number of mechanical modes to be damped. Each branch is a series composed of two inductors $\hat{L}_{i}, \tilde{L}_{i}$, a capacitor $K_{i}$ and a resistor $R_{i}$. The series $\hat{L}_{i} K_{i}$ behaves as a passband filter centred at the eigenfrequency $\omega_{i}$ of the $i$-th mechanical mode; in that frequency interval, the series $\tilde{L}_{i} R_{i}$ operates in a similar way as in a resistive-inductive single resonant circuit. Ideally, with this configuration each branch controls a single mechanical mode, without affecting the others. With this assumption, the optimal inductance for $i$-th mode would have the following expression

$$
L_{i}=\hat{L}_{i}+\tilde{L}_{i}=\frac{1}{\omega_{i}^{2}}\left(\frac{1}{K_{i}}+\frac{1}{C_{h}}\right)
$$

where $C_{h}$ is the capacitance of the piezoelectric transducer. In practice, to account for the undesired cross influence of the branches on the mechanical modes to be controlled, a further numerical fine-tuning is applied. Also, the additional capacitances $K_{i}$ 's are set approximately to $10 \%$ of the piezoelectric capacitance $C_{h}$, considering that additional capacitances worsen the electro-mechanical coupling and smaller capacitances requires larger inductances.

The performance of the proposed shunting controls is compared with this multi-modal shunting technique. To make these methods comparable, the single-shunt approach is generalised for the use of multiple piezoelectric patches. The five piezoelectric transducers are shunted with five identical multi-resonant current flowing circuits. Table 7 reports the corresponding numerical values for the circuital components in Fig. 16. The inductances of each shunt are chosen according to (C-1), with a further numerical fine-tuning. The resistances $R_{i}$ 's are optimised numerically to minimise the maximum amplitude of the plate mobility function around each natural frequency. 


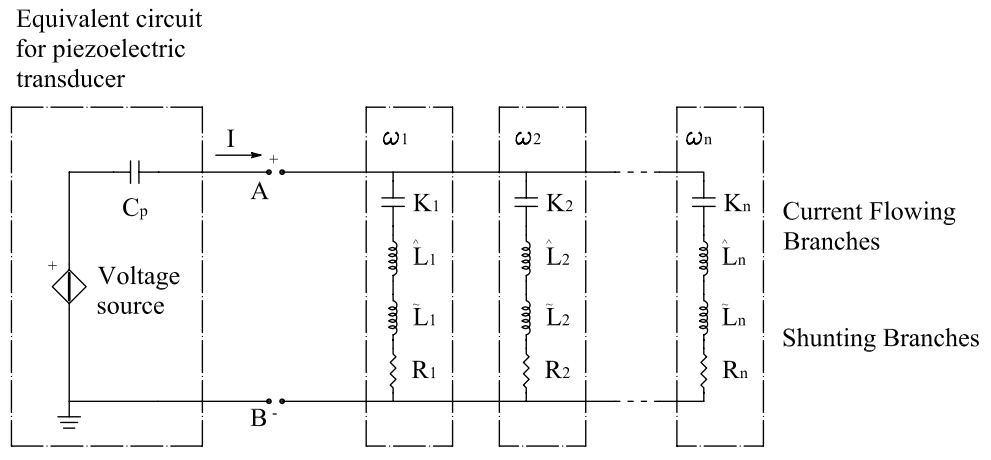

Fig. 16 A single shunt for multi-modal current flowing control.

\section{References}

1. J. Dosch, D. Inman, and E. Garcia. A self-sensing piezoelectric actuator for collocated control. Journal of Intelligent Material Systems and Structures, 3:166-185, 1992.

2. E. H. Anderson and N. W. Hagood. Simultaneous piezoelectric sensing/ actuation: analysis and application to controlled structures. Journal of Sound and Vibration, 174:617-639, 1994.

3. J. J. Hollkamp and T. F. Starchville. A self-tuning piezoelectric vibration absorber. Journal of Intelligent Material Systems and Structures, 5:559-566, 1994.

4. S. Y. Wu. Method for multiple-mode shunt damping of structural vibration using a single pzt transducer. Smart Structures and Materials 1998: Passive Damping and Isolation, 3327:159-168, 1998.

5. S. Alessandroni, U. Andreaus, F. dell'Isola, and M. Porfiri. A passive electric controller for multimodal vibrations of thin plates. Computers and Structures, 83:1236-1250, 2005.

6. F. dell'Isola and S. Vidoli. Continuum modelling of piezoelectromechanical truss beams. Archive of Applied Mechanics, 68:1-19, 1998.

7. J. Tang and K. W. Wang. Active-passive hybrid piezoelectric networks for vibration control: comparisons and improvement. Smart Materials and Structures, 10:794-806, 2001.

8. O. Thorp, M. Ruzzene, and A. Baz. Attenuation and localization of wave propagation in rods with periodic shunted piezoelectric patches. Smart Materials Structures, 10:979-989, 2001.

9. A. Badel, G. Sebald, D. Guyomar, M. Lallart, E. Lefeuvre, C. Richard, and J. Qiu. Piezoelectric vibration control by syncronized switching on adaptive voltage sources: Towards wideband semi-active damping. Journal of the Acoustical Society of America, 119(5):2815-2825, 2006.

10. N. W. Hagood and A. Von Flotow. Damping of structural vibrations with piezoelectric materials and passive electrical networks. Journal of Sound and Vibration, 146:243-268, 1991.

11. S. Y. Wu. Piezoelectric shunts with a parallel R-L circuit for structural damping and vibration control. In Proc. SPIE, Smart Materials and Structure, volume 2720, pages 259-269, 1996.

12. J. J. Hollkamp. Multimodal passive vibration suppression with piezoelectric materials and resonant shunts. Journal of Intelligent Material Systems and Structures, 5:49-57, 1994.

13. S. O. R. Moheimani, A. J. Fleming, and S. Behrens. Dynamics, stability, and control of multivariable piezoelectric shunts. IEEE/ASME Transactions on Mechatronics, 9(1):87-99, 2004.

14. U. Andreaus, F. dell'Isola, and M. Porfiri. Piezoelectric passive distributed controllers for beam flexural vibrations. Journal of Vibration and Control, 10(5):625-659, 2004.

15. C. Maurini, F. dell'Isola, and D. Del Vescovo. Comparison of piezoelectronic networks acting as distributed vibration absorbers. Mechanical Systems and Signal Processing, 18(5):1243-1271, 2004.

16. S. M. Yang and C. A. Jeng. Structural vibration suppression by concurrent piezoelectric sensor and actuator. Journal of Smart Materials and Structures, 5:806-813, 1996.

17. L. Weinberg. Network analysis and Synthesis. Mc-Graw-Hill, New York, 1962.

18. S. Tliba and H. Abou-Kandil. Modélisation et contrôle actif des vibrations d'une structure intelligente. In Actes du Ye Colloque National en Calcul des Structures, 2005.

19. C. H. Park. Dynamics modelling of beams with shunted piezoelectric elements. Journal of Sound and Vibration, 268:115-129, 2003.

20. C. Maurini, M. Porfiri, and J. Pouget. Numerical methods for modal analysis of stepped piezoelectric beams. Journal of Sound and Vibration, 298:918-933, 2006.

21. S. Behrens, S. O. R. Moheimani, and A. J. Fleming. Multiple mode current flowing passive piezoelectric shunt controller. Journal of Sound and Vibration, 266:929-942, 2003.

22. M. Porfiri, C. Maurini, and J. Pouget. Identification of electromechanical modal parameters of linear piezoelectric structures. Smart Materials and Structures, 16:323-331, 2007.

23. J. N. Juang and M. Phan. Robust controller design for second-order dynamic systems: a virtual passive approach. Journal of Guidance, Control and Dynamics, 15:1192-1198, 1992. 